\title{
Jumping coefficients and spectrum of a hyperplane arrangement
}

\author{
Nero Budur and Morihiko Saito
}

\begin{abstract}
In an earlier version of this paper written by the second named author, we showed that the jumping coefficients of a hyperplane arrangement depend only on the combinatorial data of the arrangement as conjectured by Mustaţă. For this we proved a similar assertion on the spectrum. After this first proof was written, the first named author found a more conceptual proof using the Hirzebruch-Riemann-Roch theorem where the assertion on the jumping numbers was proved without reducing to that for the spectrum. In this paper we improve these methods and show that the jumping numbers and the spectrum are calculable in low dimensions without using a computer. In the reduced case we show that these depend only on fewer combinatorial data, and give completely explicit combinatorial formulas for the jumping coefficients and (part of) the spectrum in the case the ambient dimension is 3 or 4 . We also give an analogue of Mustaţă's formula for the spectrum.
\end{abstract}

\section{Introduction}

This paper combines and improves two unpublished preprints: [29] which gave the first proof of Theorem 1 below, and [5] which gave a formula for the spectrum using the Hirzebruch-Riemann-Roch theorem [19] together with the combinatorial description of the cohomology ring of the wonderful model [8].

Let $D$ be a hyperplane arrangement in $X=\mathbf{C}^{n}$ with $D_{i}(i \in \Lambda)$ the irreducible components. In this paper we assume $D$ is central and essential (i.e. $0 \in D_{i}$ for any $i$, and $\left.\bigcap_{i} D_{i}=\{0\}\right)$ since the multiplier ideals and the spectrum are defined locally. We also assume that $D$ is not a divisor with normal crossings (i.e. $\operatorname{deg} D_{\text {red }}>n$ ). However, we do not assume $D$ is reduced.

For a positive rational number $\alpha$, the multiplier ideal $\mathcal{J}(X, \alpha D)$ is a coherent ideal of the structure sheaf $\mathcal{O}_{X}$ defined by the local integrability of $|g|^{2} /|f|^{2 \alpha}$ for $g \in \mathcal{O}_{X}$, where $f$ is a defining polynomial of $D$, see [21], [23]. This gives a decreasing sequence of ideals $\mathcal{J}(X, \alpha D)$ which is locally constant outside a locally finite subset $\mathrm{JC}(D)$ of $\mathbf{Q}$. The elements of $\mathrm{JC}(D)$ are called the jumping coefficients. Here we may restrict to the intersection with the interval $(0,1)$ since $1 \in \mathrm{JC}(D)$ and for $\alpha>0$, we have $\alpha \in \mathrm{JC}(D)$ if and only if $\alpha+1 \in \mathrm{JC}(D)$. A formula for the multiplier ideals of a hyperplane arrangement

Date: Aug. 26, 2009, v.3

The first author is supported by the NSF grant DMS-0700360.

The second author is partially supported by Kakenhi 19540023. 
$D$ was obtained by Mustaţă [22]. It was conjectured there that $\operatorname{JC}(D)$ depends only on the combinatorial data of a hyperplane arrangement.

For simplicity, consider the case where $n=3, D$ is reduced, and mult ${ }_{x} D \leq 3$ for any $x \neq 0$. Let $D^{\text {nnc }} \subset D$ denote the complement of the subset consisting of normal crossing singularities. This is the union of the lines of multiplicity 3 , and corresponds to a finite subset $\mathbf{P}\left(D^{\text {nnc }}\right) \subset \mathbf{P}^{2}$. Let $\mathbf{C}[x, y, z]_{i}$ denote the vector space of homogeneous polynomials of degree $i$. Set $d=\operatorname{deg} D$. In this case Mustaţă's formula implies the following (see [22], Cor. 2.1): If $\alpha=j / d \in\left(\frac{2}{3}, 1\right)$ with $j \in \mathbf{Z}$, then

$$
\alpha \in \mathrm{JC}(D) \Longleftrightarrow \exists g \in \mathbf{C}[x, y, z]_{j-3} \backslash\{0\} \text { with } g^{-1}(0) \supset \mathbf{P}\left(D^{\mathrm{nnc}}\right) .
$$

The last condition may a priori depend on the position of $\mathbf{P}\left(D^{\mathrm{nnc}}\right)$, and the conjecture is rather nontrivial. It turns out, however, that the points of $\mathbf{P}\left(D^{\text {nnc }}\right)$ are always in a generic position as far as the above condition is concerned, see a remark on the surjectivity of (0.2) after Theorem 5 below. Note that there is an example such that $\frac{j}{d} \notin \mathrm{JC}(D)$, see Example (3.6) below. (This implies a negative answer to [22], Question 2.7 even in the case $j=5$.)

Using an inductive argument, we gave in [29] the first proof of the following.

Theorem 1. The jumping coefficients and the spectrum of a hyperplane arrangement depend only on the combinatorial data.

The spectrum $\operatorname{Sp}(f)$ of a hypersurface singularity was defined by J. Steenbrink [31] using the monodromy and the Hodge filtration on the Milnor cohomology where $f$ is a function defining locally $D$. The spectrum $\operatorname{Sp}(f)$ is a fractional polynomial $\sum_{\alpha} n_{f, \alpha} t^{\alpha}$ where $n_{f, \alpha}=0$ for $\alpha \notin \mathbf{Q} \cap(0, n)$ (see [6], Prop. 5.2), and $\alpha$ is called a nontrivial exponent if $n_{f, \alpha} \neq 0$. It was shown in [3] that, for $\alpha \in(0,1)$, it is a jumping coefficient if it is a nontrivial exponent, and the converse holds in the isolated singularity case. Using [6], we can show for any holomorphic function $f$ that the converse also holds if $\alpha$ is an isolated jumping coefficient (i.e. if $\alpha$ is not a jumping coefficient for $D \backslash\{0\}$ ), see Proposition (4.2) below.

Returning to the case of an essential central hyperplane arrangement, let $g_{i}$ be the defining linear function of $D_{i}$ with $m_{i}$ the multiplicity. Let $V$ be an intersection of $D_{i}$ which is called an edge. Set $f_{X / V}=\prod_{D_{i} \supset V} g_{i}^{m_{i}}$. This is viewed as a function on $X / V$, and defines a hyperplane arrangement $D_{V} \subset X / V$. Let $D^{\text {nrnc }} \subset D$ denote the complement of the subset consisting of reduced normal crossing singularities. By Proposition (4.2) we then get the following.

Proposition 1. For $\alpha \in(0,1)$, we have $\alpha \in \mathrm{JC}(D)$ if and only if there is an edge $V \subset D^{\text {nrnc }}$ with $n_{f_{X / V}, \alpha} \neq 0$.

So the assertion of Theorem 1 for the jumping coefficients is reduced to the corresponding assertion for the spectrum since the combinatorial data of $D_{V} \subset X / V$ are obtained by restricting those of $D$. The spectrum of a hyperplane arrangement is calculated by using the canonical embedded resolution together with the filtered logarithmic complexes associated to certain local systems as in [16]. The assertion is then reduced to the calculation of the restriction of the de Rham complex to the exceptional divisors of the canonical 
embedded resolution where we need some arguments as in [8]. Note that the moduli space of hyperplane arrangements with fixed combinatorial data is not necessarily connected as shown in [25] as a corollary of a deep theorem (see also [27], 5.7 for a direct argument), and hence we cannot prove Theorem 1 by simply using a deformation argument.

After an earlier version of the above proof of Theorem 1 due to the second named author [29] was written, the first named author ([4], [5]) found a more conceptual proof using the Hirzebruch-Riemann-Roch theorem and a combinatorial description [8] of the cohomology ring of the canonical embedded resolution of the corresponding hyperplane arrangement in $\mathbf{P}^{n-1}$. The new proof implies formulas for the jumping coefficients and the spectrum in terms of the combinatorial data where the assertion for the jumping numbers is proved without using Proposition 1 (although it needs a compactification of $X=\mathbf{C}^{n}$ so that the calculation becomes more complicated than the proof using Proposition 1). It is also possible to write down a formula for the spectrum as in [5] by summarizing the arguments in (5.3-4) in this paper. (Note that [4] did not deal with the whole spectrum.) Stimulated by this new proof, there was an improvement of the inductive argument. Using these we can prove Theorem 2 below.

Let $\mathcal{S}(D)^{\mathrm{nnc}}$ denote the set of edges $V$ contained in $D^{\mathrm{nnc}}$. Let $\subset, \mu(V)$, and $\gamma(V)$ respectively denote the inclusion relation, multiplicity of $D$ along $V$, and the codimension of $V$, see (1.1) below. Then we have the following.

Theorem 2. Assume $D$ is reduced. Then the jumping coefficients and the coefficients $n_{f, \alpha}$ of the spectrum for $\alpha \in(0,1] \cup(n-1, n)$ depend only on the weak combinatorial equivalence class, i.e. on the set $\mathcal{S}(D)^{\mathrm{nnc}}$ together with the combinatorial data $\subset, \mu, \gamma$. For $\alpha \in(1, n-1], n_{f, \alpha}$ depends only on $\mathcal{S}(D)^{\mathrm{nnc}}$ together with $\subset, \mu, \gamma$ and also the subsets $\mathcal{S}^{D_{i}}:=\left\{V \in \mathcal{S}(D)^{\mathrm{nnc}} \mid V \subset D_{i}\right\}(i \in \Lambda)$.

The weak combinatorial equivalence is strictly weaker than the usual combinatorial equivalence. Indeed, if $n=3$, the former is determined only by $d=\operatorname{deg} D$ and

$$
\nu_{m}^{(2)}=\#\left\{y \in \mathbf{P}(D) \mid \operatorname{mult}_{y} \mathbf{P}(D)=m\right\} \quad \text { for } m \geq 3
$$

where $\mathbf{P}(D) \subset \mathbf{P}^{2}$, see also (1.1.3). For instance, in the case where $d=7, \nu_{3}^{(2)}=3$, and $\nu_{m}^{(2)}=0(m>3)$, there are two possibilities of combinatorial data depending on whether the three points of multiplicity 3 are on a same line or not.

In this paper we also show that the jumping coefficients and the spectrum of hyperplane arrangements are calculable in low dimensions without using a computer as in [4], [5] (although the formula is rather complicated). This was partly made possible by restricting the centers of the blow-ups to those contained in $D^{\text {nnc }}$. For instance we have the following.

Theorem 3. Assume $D$ reduced and $n=3$. Then $n_{f, \alpha}=0$ if $\alpha d \notin \mathbf{Z}$, and we have for $\alpha=\frac{i}{d} \in(0,1]$ with $i \in[1, d] \cap \mathbf{Z}$

$$
\begin{aligned}
n_{f, \alpha} & =\left(\begin{array}{c}
i-1 \\
2
\end{array}\right)-\sum_{m} \nu_{m}^{(2)}\left(\begin{array}{c}
\lceil i m / d\rceil-1 \\
2
\end{array}\right), \\
n_{f, \alpha+1} & =(i-1)(d-i-1)-\sum_{m} \nu_{m}^{(2)}(\lceil i m / d\rceil-1)(m-\lceil i m / d\rceil), \\
n_{f, \alpha+2} & =\left(\begin{array}{c}
d-i-1 \\
2
\end{array}\right)-\sum_{m} \nu_{m}^{(2)}\left(\begin{array}{c}
m-\lceil i m / d\rceil \\
2
\end{array}\right)-\delta_{i, d}
\end{aligned}
$$


where $\lceil\beta\rceil:=\min \{k \in \mathbf{Z} \mid k \geq \beta\}$, and $\delta_{i, d}=1$ if $i=d$ and 0 otherwise.

Theorem 4. Assume $D$ reduced and $n=4$. Let $\nu_{m}^{(2)}, \nu_{m^{\prime}}^{(3)}, \nu_{m, m^{\prime}}^{(2,3)}$ be as in (1.1.3) below. Then $n_{f, \alpha}=0$ for $\alpha d \notin \mathbf{Z}$, and we have for $\alpha=\frac{i}{d} \in(0,1]$ with $i \in[1, d] \cap \mathbf{Z}$

$$
\begin{aligned}
n_{f, \alpha} & =\left(\begin{array}{c}
i-1 \\
3
\end{array}\right)-\sum_{m, m^{\prime}} \nu_{m, m^{\prime}}^{(2,3)}\left(2\left(\begin{array}{c}
\lceil i m / d\rceil-1 \\
3
\end{array}\right)-\left(\begin{array}{c}
\lceil i m / d\rceil-1 \\
2
\end{array}\right)\left(\left\lceil i m^{\prime} / d\right\rceil-3\right)\right) \\
& +\sum_{m} \nu_{m}^{(2)}\left(2\left(\begin{array}{c}
\lceil i m / d\rceil-1 \\
3
\end{array}\right)-(i-3)\left(\begin{array}{c}
\lceil i m / d\rceil-1 \\
2
\end{array}\right)\right)-\sum_{m^{\prime}} \nu_{m^{\prime}}^{(3)}\left(\begin{array}{c}
\left\lceil i m^{\prime} / d\right\rceil-1 \\
3
\end{array}\right) .
\end{aligned}
$$

The formula is similar for $n=4$ and $\alpha \in(3,4)$. However, it requires some more combinatorial data, and is more complicated for $n=4$ and $\alpha \in(1,3]$. Those are left to the reader as exercises. Note that the formula for $n=3$ and $\alpha \in(0,1]$ is quite similar to a formula for the Hodge number in [15], Th. 6. As for the jumping coefficients, it is well-known that $\operatorname{JC}(D) \cap(0,1)=\{i / d \mid i \in \mathbf{Z} \cap[2, d)\}$ with $d=\operatorname{deg} D$ if $n=2$ and $D$ is reduced. Combined with Proposition 1, Theorems 3-4 then imply the following.

Corollary 1. Assume $D$ reduced and $n=3$. Then $\alpha \in(0,1)$ is a jumping coefficient of $D$ if and only if there is $m \in \mathbf{N} \cap[3, \infty)$ such that $m \alpha \in \mathbf{Z} \cap[2, m)$ and $\nu_{m}^{(2)} \neq 0$ or there $i s i \in \mathbf{Z} \cap[3, d)$ such that $\alpha=\frac{i}{d}$ and $n_{f, \alpha} \neq 0$ in Theorem 3 .

Corollary 2. Assume $D$ reduced and $n=4$. In the notation of (1.1.3), $\alpha \in(0,1)$ is a jumping coefficient of $D$ if and only if there is $m \in \mathbf{N} \cap[3, \infty)$ such that $m \alpha \in \mathbf{Z} \cap[2, m)$ and $\nu_{m}^{(2)} \neq 0$, or there is $V \in \mathcal{S}(D)^{\mathrm{nnc}}$ together with $i \in \mathbf{Z} \cap[3, \mu(V))$ such that $\operatorname{codim} V=3$, $\alpha=i / \mu(V)$ and $n_{f_{X / V}, \alpha} \neq 0$ in Theorem 3 , or there $i s i \in \mathbf{Z} \cap[4, d)$ such that $\alpha=i / d$ and $n_{f, \alpha} \neq 0$ in Theorem 4 . Here $\mu(V)=$ mult $_{V} D$.

We have a formula for the spectrum analogous to Mustaţă's formula [22] as follows.

Theorem 5. With the notation of (1.1) below, let $\mathcal{I}_{V} \subset \mathbf{C}[X]$ denote the reduced ideal of $V \in \mathcal{S}^{\prime}:=\mathcal{S}(D)^{\mathrm{nrnc}}$. For $\alpha=\frac{j}{d} \in(0,1]$ with $j \in[1, d] \cap \mathbf{Z}$ we have

$$
n_{f, \alpha}=\operatorname{dim}\left(\bigcap_{V \in \mathcal{S}^{\prime} \backslash\{0\}} \mathcal{I}_{V}^{\lceil\alpha \mu(V)\rceil-\gamma(V)} \cap \mathbf{C}[X]_{j-n}\right) .
$$

By Proposition (4.2) below this is compatible with Mustaţă's formula [22] for $\alpha \in(0,1)$ in the case $\mu(V) \alpha \notin \mathbf{Z}$ for any $V \in \mathcal{S}(D) \backslash\{0\}$, see also (3.3) below. Here $\mathbf{C}[X]_{j-n}$ denotes the space of homogeneous polynomials of degree $j-n$, and the intersection with $\mathcal{I}_{V}^{e_{V}}$ gives a restriction condition for $g \in \mathbf{C}[X]_{j-n}$ as in the right-hand side of (0.1) where $e_{V}=\lceil\alpha \mu(V)\rceil-\gamma(V)$. In the case $n=3$ and $D$ is reduced, this restriction condition is given by the condition that $g \in \mathbf{m}_{V}^{e_{V}}$, where $g$ is viewed as a section of $\mathcal{O}_{\mathbf{P}^{2}}(j-3)$ and $\mathbf{m}_{V} \subset \mathcal{O}_{\mathbf{P}^{2}, y}$ is the maximal ideal with $y$ the closed point of $\mathbf{P}^{2}$ corresponding to $V$. (Here $\mathcal{O}_{\mathbf{P}^{2}}(j-3)$ is locally trivialized.) Since $\operatorname{dim} \mathcal{O}_{\mathbf{P}^{2}, y} / \mathbf{m}_{V}^{e_{V}}=\left(\begin{array}{c}e_{V}+1 \\ 2\end{array}\right)$, the first equality of Theorem 3 for $\alpha \in(0,1]$ implies the surjectivity of

$$
H^{0}\left(\mathbf{P}^{2}, \mathcal{O}_{\mathbf{P}^{2}}(j-3)\right) \rightarrow \bigoplus_{V \in \mathcal{S}^{\prime} \backslash\{0\}} \mathcal{O}_{\mathbf{P}^{2}, y} / \mathbf{m}_{V}^{e_{V}}
$$

which means that the above restriction conditions for $V \in \mathcal{S}^{\prime} \backslash\{0\}$ are always independent so that they give the maximal restriction condition in total. This is closely related to 
the non-degeneracy of the matrix after (3.6.1). Note also that the above surjectivity is equivalent to the vanishing

$$
H^{1}\left(\mathbf{P}^{2}, \mathcal{O}_{\mathbf{P}^{2}}(i-3) \otimes_{\mathcal{O}} \mathcal{I}(\alpha)\right)=0
$$

where $\mathcal{I}(\alpha):=\operatorname{Ker}\left(\mathcal{O}_{\mathbf{P}^{2}} \rightarrow \bigoplus_{V \in \mathcal{S}^{\prime} \backslash\{0\}} \mathcal{O}_{\mathbf{P}^{2}, y} / \mathbf{m}_{V}^{e_{V}}\right)$.

It is known that the jumping coefficients are closely related to the roots of the $b$ function, see [14] and (3.5) below. For the moment it is unclear whether an analogue of Theorem 1 holds for the $b$-function. As for the relation with topology, note that the spectrum does not determine each Betti number of the Milnor fiber of an hyperplane arrangement, see [7], Ex. 5.4-5 and [12], p. 213, Ex. 4.16.

We would like to thank M. Mustaţă and A. Dimca for useful comments concerning this paper. We also thank the referee for useful remarks.

In Section 1, we review some facts related to hyperplane arrangements, spectrum and jumping coefficients. In Section 2, we essentially reproduce Section 2 of [29] on the canonical embedded resolution of a projective hyperplane arrangement, see also [8]. In Section 3, we prove Theorems 3 and 5. In Section 4, we give an improved version of the first proof of Theorem 1 together with proofs of Theorems $2-4$ by induction. In Section 5 , we improve some arguments in [4], [5] (using $D^{\mathrm{nnc}}$ ), and give proofs of Theorems 1-4 using the Hirzebruch-Riemann-Roch theorem and the combinatorial description of the cohomology ring of the embedded resolution.

\section{Preliminaries}

1.1. Hyperplane arrangements. Let $D$ be a central hyperplane arrangement in $X=\mathbf{C}^{n}$ with $D_{i}(i \in \Lambda)$ the irreducible components of $D$, where central means that all the $D_{i}$ pass through the origin, see [24]. We also assume $D$ is essential, and is not a divisor with normal crossings, i.e. $\bigcap_{i} D_{i}=\{0\}$ and $\operatorname{deg} D_{\text {red }}>n$. We define a set of vector subspaces of $X$ by

$$
\mathcal{S}(D)=\left\{\bigcap_{i \in I} D_{i}\right\}_{I \subset \Lambda, I \neq \emptyset}
$$

where $I$ runs over the nonempty subsets of $\Lambda$. (Note that we may have $\bigcap_{i \in I} D_{i}=\bigcap_{i \in I^{\prime}} D_{i}$ with $I \neq I^{\prime}$.) For $V \in \mathcal{S}(D)$, define

$$
I(V)=\left\{i \in \Lambda \mid D_{i} \supset V\right\}
$$

so that $V=\bigcap_{i \in I(V)} V_{i}$. There are functions $\gamma, \mu, \mu_{\text {red }}: \mathcal{S}(D) \rightarrow \mathbf{N}$ such that for $V \in \mathcal{S}(D)$

$$
\begin{aligned}
\gamma(V) & :=\operatorname{codim}_{X} V=\min \left\{|I| \mid \bigcap_{i \in I} D_{i}=V\right\}, \\
\mu(V) & :=\operatorname{mult}_{V} D=\sum_{i \in I(V)} \mu\left(D_{i}\right), \\
\mu_{\text {red }}(V) & :=\operatorname{mult}_{V} D_{\text {red }}=\# I(V) .
\end{aligned}
$$

There is a natural order on $\mathcal{S}(D)$ defined by the inclusion relation $\subset$. 
Let $D^{\text {nnc }} \subset D$ denote the complement of the subset consisting of normal crossing singularities. Here "normal crossing" means that the associated reduced variety has normal crossings. We define similarly $D^{\text {nrnc }}$ by replacing "normal crossing" with "reduced normal crossing" so that $D^{\text {nnc }} \subset D^{\text {nrnc }}$. (In this paper we do not assume $D^{\text {nnc }} \neq \emptyset$.) Set

$$
\left.\mathcal{S}(D)^{\mathrm{nnc}}=\left\{V \in \mathcal{S}(D) \mid V \subset D^{\mathrm{nnc}}\right\} \text { (similarly for } \mathcal{S}(D)^{\mathrm{nrnc}}\right) .
$$

In this paper $D^{\text {nrnc }}$ is used only in Theorem 5 and Mustaţă's formula, see (3.3-4).

For $\mathcal{S}:=\mathcal{S}(D)^{\mathrm{nnc}}$, set

$$
\begin{aligned}
& \mathcal{S}^{D_{i}}=\left\{V \in \mathcal{S} \mid V \subset D_{i}\right\}, \quad \mathcal{S}^{(i)}=\{V \in \mathcal{S} \mid \gamma(V)=i\}, \\
& \nu_{m}^{(i)}=\#\left\{V \in \mathcal{S}^{(i)} \mid \mu(V)=m\right\}, \\
& \nu_{m, m^{\prime}}^{(i, j)}=\#\left\{\left(V, V^{\prime}\right) \in \mathcal{S}^{(i)} \times \mathcal{S}^{(j)} \mid V \supset V^{\prime}, \mu(V)=m, \mu\left(V^{\prime}\right)=m^{\prime}\right\} .
\end{aligned}
$$

This is compatible with the definition of $\nu_{m}^{(2)}$ in Introduction.

1.2. Combinatorial equivalence class. With the above notation we call

$$
\mathcal{S}(D), \subset, \mu
$$

the (strong) combinatorial data of a hyperplane arrangement $D$. Note that $\gamma$ is determined by the inclusion relation $C$, and so is $\mu$ in the case $D$ is reduced. We call

$$
\mathcal{S}(D)^{\mathrm{nnc}}, \subset, \mu, \gamma
$$

the weak combinatorial data. We say that two hyperplane arrangements $D$ and $D^{\prime}$ are combinatorially equivalent if there is a one-to-one correspondence between $\mathcal{S}(D)$ and $\mathcal{S}\left(D^{\prime}\right)$ in a compatible way with $\subset, \mu$ (similarly for weak combinatorial equivalence).

1.3. Milnor fiber and the covering. In this subsection $D \subset X:=\mathbf{C}^{n}$ is defined by a homogeneous polynomial $f$. Set $Z=\mathbf{P}(D)$. This is a subvariety of $Y:=\mathbf{P}^{n-1}$ defined by $f$. There is a ramified covering

$$
Y^{\prime}:=\operatorname{Spec}_{Y}\left(\bigoplus_{0 \leq k<d} \mathcal{S}^{k}\right) \stackrel{\pi}{\rightarrow} Y,
$$

where $\mathcal{S}^{k}=\mathcal{O}_{Y}(-k)$ and $f$ induces morphisms $\mathcal{O}_{Y}(-k-d) \rightarrow \mathcal{O}_{Y}(-k)$ defining a ring structure on $\bigoplus_{0 \leq k<d} \mathcal{S}^{k}$.

Let $U=Y \backslash Z$, and $U^{\prime}$ be the restriction of $Y^{\prime}$ over $U$. Then $U^{\prime}$ is étale over $U$, and the Milnor fiber $f^{-1}(1)$ is identified with $U^{\prime}$. Indeed, $Y^{\prime}$ is identified with a section of the line bundle over $Y$ corresponding to $\mathcal{O}_{Y}(1)$, and $U^{\prime}$ is identified with a section of its dual bundle which is isomorphic to the blow-up of $\mathbf{C}^{n}$ at the origin. So $U^{\prime}$ is identified with the divisor on $\mathbf{C}^{n}$ defined by $f=1$.

The geometric Milnor monodromy is induced by an automorphism of $\mathbf{C}^{n}$ defined by

$$
T_{g}:\left(x_{1}, \ldots, x_{n}\right) \mapsto\left(\xi x_{1}, \ldots, \xi x_{n}\right),
$$


where $\xi=\exp (2 \pi \sqrt{-1} / d)$. Note that the monodromy of the cohomology local system associated with the Milnor fibration on a punctured disk is given by $\left(T_{g}^{*}\right)^{-1}$. It is wellknown (see e.g. [7], [12]) that

$$
\mathcal{S}^{k}=\operatorname{Ker}\left(\left(T_{g}^{*}\right)^{-1}-\xi^{k}\right) \subset \pi_{*} \mathcal{O}_{Y^{\prime}}=\bigoplus_{0 \leq k<d} \mathcal{S}^{k} .
$$

For the convenience of the reader, we include here a proof. Using the projective coordinates $z_{0}, \ldots, z_{n}$ of $\mathbf{P}^{n} \supset \mathbf{C}^{n}$ such that $x_{i}=z_{i} / z_{0}$ for $i \in[1, n]$, the geometric monodromy is induced by

$$
T_{g}:\left(z_{0}, z_{1}, \ldots, z_{n}\right) \mapsto\left(\xi^{-1} z_{0}, z_{1}, \ldots, z_{n}\right) .
$$

After changing the order of the coordinates $z_{1}, \ldots, z_{n}$ if necessary, let $y_{i}=z_{i} / z_{n}$ on $\left\{z_{n} \neq 0\right\} \subset \mathbf{P}^{n}$ for $i \in[0, n-1]$. Set $h\left(y_{0}, \ldots, y_{n-1}\right)=f\left(z_{1}, \ldots, z_{n}\right) / z_{n}^{d}$. Then $Y^{\prime} \subset \mathbf{P}^{n}$ is locally defined by the equations

$$
f\left(x_{1}, \ldots, x_{n}\right)=1, \quad f\left(z_{1}, \ldots, z_{n}\right)=z_{0}^{d}, \quad h\left(y_{0}, \ldots, y_{n-1}\right)=y_{0}^{d},
$$

and the restriction of $\pi_{*} \mathcal{O}_{Y^{\prime}}$ to $\mathbf{C}^{n-1}=\left\{z_{n} \neq 0\right\} \subset \mathbf{P}^{n-1}$ is identified with

$$
\pi_{*}\left(\mathcal{O}_{\mathbf{C}^{n}} /\left(y_{0}^{d}-h\left(y_{0}, \ldots, y_{n-1}\right)\right)=\bigoplus_{k=0}^{d-1} \mathcal{O}_{\mathbf{C}^{n-1}} y_{0}^{k},\right.
$$

where $\left\{z_{n} \neq 0\right\} \subset \mathbf{P}^{n}$ is identified with $\mathbf{C}^{n}$. On the other hand, the action of $T_{g}^{*}$ on the coordinate $y_{i}$ is the multiplication by $\xi^{-1}$ for $i=0$, and the identity for $i \neq 0$. So (1.3.1) follows.

1.4. Spectrum. With the notation of (1.3) the spectrum $\operatorname{Sp}(f)=\sum_{\alpha \in \mathbf{Q}} n_{f, \alpha} t^{\alpha}$ is defined by

$$
\begin{gathered}
n_{f, \alpha}=\sum_{j}(-1)^{j-n+1} \operatorname{dim} \operatorname{Gr}_{F}^{p} \widetilde{H}^{j}\left(f^{-1}(1), \mathbf{C}\right)_{\lambda} \\
\text { with } p=\lfloor n-\alpha\rfloor, \lambda=\exp (-2 \pi i \alpha),
\end{gathered}
$$

where $\widetilde{H}^{j}\left(f^{-1}(1), \mathbf{C}\right)_{\lambda}$ is the $\lambda$-eigenspace of the reduced cohomology of $f^{-1}(1) \subset \mathbf{C}^{n}$ for the semi-simple part of the Milnor monodromy, and $F$ is the Hodge filtration, see [31]. Here $\lfloor\beta\rfloor:=\max \{k \in \mathbf{Z} \mid k \leq \beta\}$. By [6], Prop. 5.2, we have

$$
n_{f, \alpha}=0 \quad \text { if } \quad \alpha \notin(0, n) .
$$

By (1.3.1) $\mathcal{S}^{k}$ has a meromorphic connection with regular singularities along $Z$, and hence the localization $\mathcal{S}^{k}(* Z)$ along $Z$ is a regular holonomic $\mathcal{D}_{Y}$-module, which is locally isomorphic to $\mathcal{O}_{Y}(* Z) h^{k / d}$ where $h$ is as in (1.3). (This is proved by using the equation $h=y_{0}^{d}$.) Moreover we get by (1.3.1)

$$
H^{j}\left(U, \operatorname{DR}\left(\left.\mathcal{S}^{k}\right|_{U}\right)\right)=H^{j}\left(f^{-1}(1), \mathbf{C}\right)_{\lambda} \quad \text { with } \lambda=\exp (2 \pi i k / d) .
$$

Since $\mathcal{S}^{k}$ has rank 1, this implies

$$
\sum_{j \in \mathbf{Z}}(-1)^{j} \operatorname{dim} H^{j}\left(f^{-1}(1), \mathbf{C}\right)_{\lambda}=\chi(U) .
$$


Let $\rho: \widetilde{Y} \rightarrow Y$ be an embedded resolution of $Z$ inducing an isomorphism over $Y \backslash Z$. We have a divisor with normal crossings on $\widetilde{Y}$

$$
\widetilde{Z}:=\rho^{*} Z=Z^{\prime}+Z^{\prime \prime} \quad \text { with } Z^{\prime}=\sum_{j \in J^{\prime}} m_{j} E_{j}, Z^{\prime \prime}=\sum_{j \in J^{\prime \prime}} m_{j} E_{j},
$$

where $Z^{\prime}$ is the proper transform of $Z, Z^{\prime \prime}$ is the exceptional divisor, and the $E_{j}$ are the irreducible components with multiplicity $m_{j}$. Set $J=J^{\prime} \cup J^{\prime \prime}$.

Let $\widetilde{\mathcal{S}}^{k}$ be the Deligne extension of $\left.\mathcal{S}^{k}\right|_{U}$ over $\widetilde{Y}$ such that the eigenvalues of the residue of the connection are contained in $[0,1)$, see [9]. Let $\widetilde{H}$ be the total (or proper) transform of a general hyperplane $H$ of $Y$. Using the pull-back of the above local form $\mathcal{O}_{Y}(* Z) h^{k / d}$, we get

$$
\widetilde{\mathcal{S}}^{k}=\mathcal{O}_{\widetilde{Y}}\left(-k \widetilde{H}+\sum_{j \in J}\left\lfloor k m_{j} / d\right\rfloor E_{j}\right),
$$

since $\mathcal{O}_{Y}(Z)=\mathcal{O}_{Y}(d H)$. Indeed, the eigenvalue of the residue of the connection along $E_{j}$ is

$$
k m_{j} / d-\left\lfloor k m_{j} / d\right\rfloor .
$$

Note that the above summation is taken over $J^{\prime \prime}$ in case $D$ is reduced since $\left\lfloor k m_{j} / d\right\rfloor=0$ if $m_{j}=1$ and $k \in[0, d)$. It is known (see e.g. [16]) that the associated filtered logarithmic complex together with the filtration $\sigma$ (see [10]) calculates the Hodge filtration on the cohomology. It coincides with the Hodge filtration obtained from the theory of mixed Hodge modules. (This is shown by using e.g. [26], 3.11.) So we get

$$
\operatorname{Gr}_{F}^{p} H^{p+q}\left(f^{-1}(1), \mathbf{C}\right)_{\lambda}=H^{q}\left(\widetilde{Y}, \Omega_{\widetilde{Y}}^{p}(\log \widetilde{Z}) \otimes_{\mathcal{O}} \mathcal{O}_{\widetilde{Y}}\left(-k \widetilde{H}+\sum_{j}\left\lfloor k m_{j} / d\right\rfloor E_{j}\right)\right) .
$$

Since $f^{-1}(1)$ is affine and $(n-1)$-dimensional, we have for $q>n-1-p$

$$
H^{q}\left(\widetilde{Y}, \Omega_{\widetilde{Y}}^{p}(\log \widetilde{Z}) \otimes_{\mathcal{O}} \mathcal{O}_{\widetilde{Y}}\left(-k \widetilde{H}+\sum_{j \in J}\left\lfloor k m_{j} / d\right\rfloor E_{j}\right)\right)=0 .
$$

This is closely related to the Kodaira-Nakano type vanishing theorem in [16]. We also have

$$
\begin{aligned}
\Omega_{\widetilde{Y}}^{n-1}(\log \widetilde{Z}) & =\mathcal{O}_{\widetilde{Y}}\left(-n \widetilde{H}+\sum_{j \in J} c_{j} E_{j}\right), \\
& =\mathcal{O}_{\widetilde{Y}}\left((d-n) \widetilde{H}+\sum_{j \in J}\left(c_{j}-m_{j}\right) E_{j}\right),
\end{aligned}
$$

where $c_{j}$ is the codimension of the center of the blow-up corresponding to $E_{j}$.

From (1.4.3) we deduce

1.5. Proposition. For $\alpha=\frac{i}{d}+\ell$ with $i=d-k \in[1, d]$ and $\ell=n-1-p \in[0, n-1]$

$$
n_{f, \alpha}=(-1)^{\ell} \chi\left(\widetilde{Y}, \Omega_{\widetilde{Y}}^{p}(\log \widetilde{Z}) \otimes_{\mathcal{O}} \mathcal{O}_{\widetilde{Y}}\left(-k \widetilde{H}+\sum_{j \in J}\left\lfloor k m_{j} / d\right\rfloor E_{j}\right)\right) .
$$

Here $\lfloor\beta\rfloor:=\max \{k \in \mathbf{Z} \mid k \leq \beta\}$.

1.6. Compatibility with the usual definition. The above definition of the spectrum coincides with the definition using the mixed Hodge structure obtained by $H^{k} i_{0}^{*} \psi_{f} \mathbf{Q}_{X}$ 
where $\psi_{f}$ denotes the nearby cycle functor (see [11]) and $i_{0}:\{0\} \rightarrow X_{0}:=f^{-1}(0)$ denotes the inclusion. Indeed, by the compatibility of $\psi$ with the direct image under the blow-up $\rho: \widetilde{X} \rightarrow X$ at 0 (see [26], 2.14), we have isomorphisms of mixed Hodge modules

$$
{ }^{p} R^{k} \rho_{*} \psi_{\widetilde{f}}\left(\mathbf{Q}_{\widetilde{X}}[n]\right)= \begin{cases}\psi_{f}\left(\mathbf{Q}_{X}[n]\right) & \text { if } k=0 \\ 0 & \text { if } k \neq 0\end{cases}
$$

where $\psi$ is shifted by -1 so that it preserves the perverse sheaves. So we get an isomorphism in the derived category of (algebraic) mixed Hodge modules

$$
\psi_{f} \mathbf{Q}_{X}=\mathbf{R} \rho_{*} \psi_{\widetilde{f}} \mathbf{Q}_{\widetilde{X}}
$$

Note that $Y$ is identified with the exceptional divisor of the blow-up. Let $i_{Y}: Y \rightarrow \widetilde{X}_{0}:=$ $\widetilde{f}^{-1}(0)$ and $a_{Y}: Y \rightarrow p t$ denote the natural morphisms. Using the base change of $\rho$ by $i_{0}$, we then get

$$
H^{k} i_{0}^{*} \psi_{f} \mathbf{Q}_{X}=H^{k} i_{0}^{*} \mathbf{R} \rho_{*} \psi_{\widetilde{f}} \mathbf{Q}_{\widetilde{X}}=H^{k}\left(a_{Y}\right)_{*} i_{Y}^{*} \psi_{\widetilde{f}} \mathbf{Q}_{\widetilde{X}}
$$

Moreover, if $j^{\prime}: U \rightarrow \widetilde{X}_{0}$ denotes the inclusion, we have by [6], 4.2

$$
\left(i_{Y}\right)_{*} i_{Y}^{*} \psi_{\widetilde{f}} \mathbf{Q}_{\widetilde{X}}=\mathbf{R} j_{*}^{\prime} j^{\prime *} \psi_{\widetilde{f}} \mathbf{Q}_{\widetilde{X}}
$$

We get thus

$$
H^{k} i_{0}^{*} \psi_{f} \mathbf{Q}_{X}=H^{k}\left(a_{U}\right)_{*} j^{\prime *} \psi_{\widetilde{f}} \mathbf{Q}_{\widetilde{X}}
$$

So the desired compatibility is reduced to the isomorphism between the $\lambda$-eigenspace of the local system $\left.\psi_{\widetilde{f}} \mathbf{C}_{\widetilde{X}}\right|_{U}$ and the local system corresponding to $\left.\mathcal{S}^{k}\right|_{U}$ where $\lambda=$ $\exp (2 \pi \sqrt{-1} k / d)$ since the Hodge filtration is given by the filtration $\sigma_{\geq p}$ on the logarithmic de Rham complex. To show the isomorphism of local systems, it is enough to show the coincidence of the local monodromies of the two local systems along any irreducible components (indeed, this implies the triviality of the tensor product of one local system with the inverse of the other since a local system of rank 1 on $U \subset \mathbf{P}^{n-1}$ is trivial if the local monodromies are). By the calculation of the residue of the connection in (1.4), the local monodromy of $\left.\mathcal{S}^{k}\right|_{U}$ along $E_{j}$ is the multiplication by $\exp \left(-2 \pi \sqrt{-1} k m_{j} / d\right)$ where $m_{j}$ is the multiplicity of $\widetilde{f}$ along $E_{j}$. For $\lambda=\exp (2 \pi \sqrt{-1} k / d)$, it is well-known that the monodromy of the $\lambda$-eigenspace of the local system $\left.\psi_{\widetilde{f}} \mathbf{C}_{\widetilde{X}}\right|_{U}$ along $E_{j}$ is the multiplication by $\lambda^{-m_{j}}$ (since the Milnor fiber is locally defined by $x_{0}^{d} x_{j}^{m_{j}}=t$ on a neighborhood of a general point of $E_{j}$ where $x_{0}$ defines the exceptional divisor of the blow-up along $0 \in X$ ), see also [26], 3.3. So the assertion follows.

1.7. Weight filtration on the cohomology of the complement. From now on, we assume that $D$ is an essential central hyperplane arrangement. Let $U=Y \backslash \mathbf{P}(D)$ with $j_{U}: U \rightarrow Y=\mathbf{P}^{n-1}$ the inclusion. Since $U$ is affine, it is known that

$$
\left(j_{U}\right) \mathbf{Q}_{U}[n-1], \quad \mathbf{R}\left(j_{U}\right)_{*} \mathbf{Q}_{U}[n-1]
$$


are perverse sheaves (see [1]) and underlie mixed Hodge modules (see [26]). So they have the canonical weight filtration $W$ as perverse sheaves. Set

$$
\overline{\mathcal{S}}(D)=\mathcal{S}(D) \cup\{X\}, \quad \overline{\mathcal{S}}(D)^{(j)}=\{V \in \overline{\mathcal{S}}(D) \mid \gamma(V)=j\}
$$

where $\gamma(V)=\operatorname{codim} V$. Then we can show

$$
\begin{aligned}
\operatorname{Gr}_{n-1-j}^{W}\left(\left(j_{U}\right) ! \mathbf{Q}_{U}[n-1]\right) & =\bigoplus_{V \in \overline{\mathcal{S}}(D)^{(j)}} L_{V}[n-1-j], \\
\operatorname{Gr}_{n-1+j}^{W}\left(\mathbf{R}\left(j_{U}\right)_{*} \mathbf{Q}_{U}[n-1]\right) & =\bigoplus_{V \in \overline{\mathcal{S}}(D)^{(j)}} L_{V}(-j)[n-1-j],
\end{aligned}
$$

where the $L_{V}$ are polarized constant variations of Hodge structures of type $(0,0)$ on $\mathbf{P}(V) \subset \mathbf{P}^{n-1}$. It is enough to show the first isomorphism since the $L_{V}$ are self-dual by the polarization so that the second follows from the first by duality.

By increasing induction on $j \geq 0$, we define Q-vector spaces $L_{V}\left(V \in \overline{\mathcal{S}}(D)^{(j)}\right)$ together with morphisms

$$
\rho_{V, V^{\prime}}: L_{V^{\prime}} \rightarrow L_{V} \quad \text { for } V \in \overline{\mathcal{S}}(D)^{(j)}, V^{\prime} \in \overline{\mathcal{S}}(D)^{(j-1)}
$$

such that $\rho_{V, V^{\prime}}=0$ unless $V \subset V^{\prime}$ as follows: Set $L_{V}=\mathbf{Q}$ if $j=0$ or 1 , and $\rho_{V, V^{\prime}}=i d$ if $\gamma(V)=1, \gamma\left(V^{\prime}\right)=0$. Assume $L_{V}$ and $\rho_{V, V^{\prime}}$ are defined for $\gamma(V)<j$. For $V \in \overline{\mathcal{S}}(D)^{(j)}$, define

$$
\begin{aligned}
& L_{V}=\operatorname{Coker}\left(\sum \rho_{V^{\prime}, V^{\prime \prime}}: \bigoplus_{V^{\prime \prime} \in \overline{\mathcal{S}}(D)_{V}^{j-2}} L_{V^{\prime \prime}} \rightarrow \bigoplus_{V^{\prime} \in \overline{\mathcal{S}}(D)_{V}^{j-1}} L_{V^{\prime}}\right), \\
& \text { where } \overline{\mathcal{S}}(D)_{V^{\prime}}^{(j)}:=\left\{V \in \overline{\mathcal{S}}(D) \mid V \supset V^{\prime}\right\} .
\end{aligned}
$$

The morphism $\rho_{V, V^{\prime}}: L_{V^{\prime}} \rightarrow L_{V}$ for $V^{\prime} \in \overline{\mathcal{S}}(D)_{V}^{(j-1)}$ is given by the composition

$$
L_{V^{\prime}} \rightarrow \bigoplus_{V^{\prime} \in \overline{\mathcal{S}}(D)_{V}^{j-1}} L_{V^{\prime}} \rightarrow L_{V}
$$

where the first morphism is the natural inclusion, and the second is the projection to the quotient. Note that the $L_{V}$ are Hodge structures of type $(0,0)$, and they have a canonical polarization using the semi-simplicity induced by the polarization inductively.

From now on, $L_{V}$ will be identified with a constant sheaf on $\mathbf{P}(V) \subset Y=\mathbf{P}^{n-1}$ with stalk $L_{V}$. Then $\rho_{V^{\prime}, V}: L_{V^{\prime}} \rightarrow L_{V}$ is viewed as a morphism of sheaves. Define a sheaf on $Y$ by

$$
\mathcal{K}_{Y}^{j}=\bigoplus_{V \in \overline{\mathcal{S}}(D)^{(j)}} L_{V}
$$

We have the morphism $d: \mathcal{K}_{Y}^{j-1} \rightarrow \mathcal{K}_{Y}^{j}$ induced by the $\rho_{V^{\prime}, V}$, and $d \circ d=0$ by the above construction. Note that the $\mathcal{K}_{Y}^{j}[n-1-j]$ and hence $\mathcal{K}_{Y}^{\bullet}[n-1]$ are perverse sheaves on $Y$, see [1]. Then (1.7.1) is reduced to the following lemma since the weight filtration $W$ is induced by the truncation $\sigma_{\geq k}$ up to a shift.

1.8. Lemma. There is a quasi-isomorphism

$$
\left(j_{U}\right) \mathbf{Q}_{U} \stackrel{\sim}{\longrightarrow} \mathcal{K}_{Y}^{\bullet},
$$


induced by the canonical morphism $\left(j_{U}\right) ! \mathbf{Q}_{U} \rightarrow \mathbf{Q}_{Y}=\mathcal{K}_{Y}^{0}$.

Proof. Set

$$
Y^{(j)}=\bigcup_{V \in \overline{\mathcal{S}}(D))^{(j)}} \mathbf{P}(V), \quad U^{(j)}=Y^{(j)} \backslash Y^{(j+1)} .
$$

Let $\sigma_{\leq j} \mathcal{K}_{Y}^{\bullet}$ denote the quotient complex of $\mathcal{K}_{Y}^{\bullet}$ as in [10], 1.4.7, i.e. $\left(\sigma_{\leq j} \mathcal{K}_{Y}\right)^{i}=\mathcal{K}_{Y}^{i}$ if $i \leq j$, and 0 otherwise. By increasing induction on $j$ we show

$$
\left(A_{j}\right) \quad C\left(\left(j_{U}\right) ! \mathbf{Q}_{U} \rightarrow \sigma_{\leq j} \mathcal{K}_{Y}^{\bullet}\right)[n-2] \text { is a perverse sheaf supported on } Y^{(j+1)} \text {. }
$$

This is clear if $j=0$. Assume $\left(A_{j-1}\right)$ holds with $j>0$. Let $y$ be a general point of $\mathbf{P}(V)$ with $V \in \overline{\mathcal{S}}(D)^{(j)}$. Then $\left(A_{j-1}\right)$ implies

$$
H^{k}\left(\sigma_{<j} \mathcal{K}_{Y, y}^{\bullet}\right)=0 \text { for } k \neq j-1
$$

(Indeed, the restriction of any perverse sheaf to a sufficiently small Zariski-open subvariety of its support is a local system shifted by the dimension of the variety.) Moreover, by (1.7.2), we have the isomorphism as vector spaces

$$
H^{j-1}\left(\sigma_{<j} \mathcal{K}_{Y, y}^{\bullet}\right)=L_{V}
$$

and this implies the acyclicity of $\sigma_{\leq j} \mathcal{K}_{Y, y}^{\bullet}$. Since the restriction of the cohomology sheaves $\mathcal{H}^{i} \sigma_{\leq j} \mathcal{K}_{Y}^{\bullet}$ to $U^{(j)}$ are locally constant, we see that $\sigma_{\leq j} \mathcal{K}_{Y}^{\bullet}$ is acyclic on $U^{(j)}$ and hence on $Y^{(1)} \backslash Y^{(j+1)}$ using $\left(A_{j-1}\right)$ on $Y^{(1)} \backslash Y^{(j)}$ (since $\left(j_{U}\right)$ ! $\left.\left.\mathbf{Q}_{U}\right|_{Y^{(1)}}=0\right)$. So the shifted mapping cone in $\left(A_{j}\right)$ is supported on $Y^{(j+1)}$, and it remains to show that the shifted mapping cone is a perverse sheaf, i.e. in the abelian category of perverse sheaves (see [1]) we have

$$
\operatorname{Coker}\left(\left(j_{U}\right) ! \mathbf{Q}_{U}[n-1] \rightarrow\left(\sigma_{\leq j} \mathcal{K}_{Y}^{\bullet}\right)[n-1]\right)=0
$$

But this is clear since the $\mathbf{Q}_{\mathbf{P}(V)}[\operatorname{dim} \mathbf{P}(V)]$ are simple perverse sheaves so that there are no nontrivial subquotients of the perverse sheaf $\mathcal{K}_{Y}^{i}[n-1-i]$ supported on $Y^{(j+1)}$ if $i \leq j$. Thus we get $\left(A_{j}\right)$, and (1.8.1) follows by induction.

1.9. Remark. Set $r_{V}=\operatorname{rank} L_{V}$, and $\overline{\mathcal{S}}(D)_{V}:=\left\{V^{\prime} \in \overline{\mathcal{S}}(D) \mid V^{\prime} \supset V\right\}$. By Lemma (1.8) we have

$$
\sum_{V^{\prime} \in \overline{\mathcal{S}}(D)_{V}}(-1)^{\gamma\left(V^{\prime}\right)} r_{V^{\prime}}=0
$$

This implies that $(-1)^{\gamma(V)} r_{V}$ coincides with the Möbius function defined by increasing induction on $\gamma(V)$, see [24].

Take any $D_{k}$, and set

$$
\overline{\mathcal{S}}(D)_{\langle k\rangle}^{(j)}=\left\{V \in \overline{\mathcal{S}}(D)^{(j)} \mid V \not \subset D_{k}\right\}, \quad U_{k}=Y \backslash D_{k}=\mathbf{C}^{n-1},
$$

with the inclusion $j_{k}: U \rightarrow U_{k}$. Then

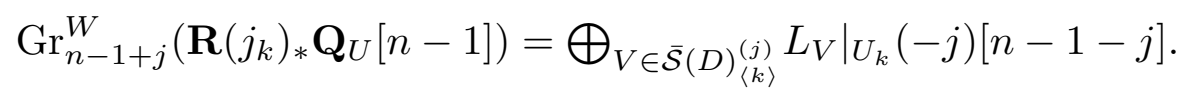


The associated spectral sequence degenerates at $E_{1}$, since the $U_{k} \cap \mathbf{P}(V)$ are affine spaces. This implies that $H^{j}(U, \mathbf{Q})$ has type $(j, j)$, and we get $F^{j} H^{j}(U, \mathbf{C})=P^{j} H^{j}(U, \mathbf{C})=$ $H^{j}(U, \mathbf{C})$ for any $j$, where $P$ is the pole order filtration. This gives examples where $P \neq F$ locally but $P=F$ globally, see [13]. The above assertion is compatible with a result of E. Brieskorn [2] that $H^{j}(U, \mathbf{Q})$ is generated by logarithmic forms

$$
\frac{d g_{i_{1}}}{g_{i_{1}}} \wedge \cdots \wedge \frac{d g_{i_{j}}}{g_{i_{j}}}
$$

where the $g_{i}$ are linear functions with constant terms defining $\mathbf{P}\left(D_{i}\right) \backslash \mathbf{P}\left(D_{k}\right) \subset \mathbf{C}^{n-1}$. The $E_{1}$-degeneration also implies a formula in [24]

$$
b_{k}(U)=\sum_{V \in \overline{\mathcal{S}}(D)_{\langle k\rangle}^{(j)}} r_{V} .
$$

It is well-known that the Betti numbers $b_{k}(U)$ are combinatorial invariants of a hyperplane arrangement, see [24]. We have a refinement as follows.

1.10. Proposition. Set $\mathcal{S}=\mathcal{S}(D)^{\mathrm{nnc}}, \mathcal{S}^{D_{i}}=\left\{V \in \mathcal{S} \mid V \subset D_{i}\right\}$ in the notation of (1.1). Then the $b_{k}(U)$ are determined by $\mathcal{S}, \subset, \mu_{\text {red }}, \gamma$ together with $\mathcal{S}^{D_{i}}(i \in \Lambda)$.

Proof. We first show that the $r_{V}$ are determined by the above combinatorial data. We have $r_{V}=1$ for $\overline{\mathcal{S}}(D) \backslash \mathcal{S}$ since $\mathcal{K}_{Y, y}^{\bullet}$ for $y \notin \mathbf{P}\left(D^{\text {nnc }}\right)$ is the standard Koszul complex. Since the $r_{V}$ for $V \in \mathcal{S}$ is determined by induction on $\gamma(V)$ using (1.9.1), it is enough to express

$$
\left|\overline{\mathcal{S}}(D)_{V}^{(j)} \backslash \mathcal{S}\right|
$$

using only the combinatorial data as above. Set $I(V)=\left\{i \in \Lambda \mid D_{i} \supset V\right\}$. Note that $I(V)$ is determined by the $\mathcal{S}^{D_{i}}(i \in \Lambda)$ if $V \in \mathcal{S}$. Set

$$
\begin{aligned}
S(\Lambda)^{(j)} & =\{I \subset \Lambda|| I \mid=j\}, \quad S(\Lambda)_{V}=\{I \subset \Lambda \mid I \subset I(V)\}, \\
S^{\text {nnc }}(\Lambda) & =\left\{I \subset \Lambda \mid I=I\left(V^{\prime}\right) \text { for some } V^{\prime} \in \mathcal{S}\right\} .
\end{aligned}
$$

Then we have the identification

$$
\overline{\mathcal{S}}(D)_{V}^{(j)} \backslash \mathcal{S}=S(\Lambda)^{(j)} \cap S(\Lambda)_{V} \backslash S^{\mathrm{nnc}}(\Lambda),
$$

and the assertion follows. Thus the $r_{V}$ are calculated by induction on $\gamma(V)$ using only the above combinatorial data.

Since the Betti number is calculated by using (1.9.2), it is enough to express

$$
\left|\overline{\mathcal{S}}(D)_{\langle k\rangle}^{(j)} \backslash \mathcal{S}\right|
$$

by using only the combinatorial data as above. So the assertion follows since

$$
\overline{\mathcal{S}}(D)_{\langle k\rangle}^{(j)} \backslash \mathcal{S}=S(\Lambda)^{(j)} \cap S(\Lambda)_{\langle k\rangle} \backslash S^{\mathrm{nnc}}(\Lambda),
$$

where $S(\Lambda)_{\langle k\rangle}=\{I \subset \Lambda \mid k \notin I\}$. This finishes the proof of Proposition (1.10). 


\section{Canonical embedded resolution}

The material in this section is treated in a much more general situation in [8]. For the convenience of the reader we treat it under the assumption that $\mathcal{S}$ is stable by intersection. This hypothesis is satisfied in our case, and simplifies certain arguments very much.

2.1. Construction. Let $\mathcal{S}$ be a finite set of proper vector subspaces of the vector space $X=\mathbf{C}^{n}$ which is stable by intersection (i.e. $V \cap V^{\prime} \in \mathcal{S}$ if $V, V^{\prime} \in \mathcal{S}$ ) and such that $0 \in \mathcal{S}$. We have a function $\gamma: \mathcal{S} \rightarrow \mathbf{N}$ associating the codimension of $V$. There is a natural order on $\mathcal{S}$ defined by the inclusion relation. We say that $\mathcal{S}$ and $\mathcal{S}^{\prime}$ are combinatorially equivalent if there is a one-to-one correspondence $\mathcal{S} \rightarrow \mathcal{S}^{\prime}$ as ordered sets in a compatible way with $\gamma$. Note that a nested subset of $\mathcal{S}$ in the sense of [8] is always linearly ordered by the inclusion relation in our paper since $\mathcal{S}$ is stable by intersection.

Let $Y=\mathbf{P}^{n-1}$. For a vector subspace $V \subset X=\mathbf{C}^{n}$, its corresponding subspace of $Y$ will be denoted by $\mathbf{P}(V)$. For $\mathcal{S}$ as above, there is a sequence of blowing-ups

$$
\rho_{i}: Y_{i+1} \rightarrow Y_{i} \text { for } 0 \leq i<n-2,
$$

whose center $C_{i}$ is the disjoint union of the proper transforms of $\mathbf{P}(V)$ for $V \in \mathcal{S}$ with $\operatorname{dim} \mathbf{P}(V)=i$, where $Y_{0}=Y$. Note that we cannot restrict to the dense edges as in [30] because this is not adequate for our inductive argument.

Set $\widetilde{Y}=Y_{n-2}$ with $\rho: \widetilde{Y} \rightarrow Y$ the composition of the $\rho_{i}$. We will sometimes denote $\tilde{Y}$ by $Y^{\mathcal{S}}$. This applies to $\mathbf{P}(V)^{\mathcal{S}^{V}}$ where $Y$ and $\mathcal{S}$ are replaced by $\mathbf{P}(V)$ and $\mathcal{S}^{V}$ respectively. Here we define for $V \in \mathcal{S}$

$$
\mathcal{S}^{V}=\left\{V^{\prime} \in \mathcal{S} \mid V^{\prime} \varsubsetneqq V\right\}, \quad \mathcal{S}_{V}=\left\{V^{\prime} \in \mathcal{S} \mid V^{\prime} \supset V\right\} .
$$

For $V \in \mathcal{S} \backslash\{0\}$ with $\operatorname{dim} \mathbf{P}(V)=i$, let $C_{V, j}$ denote the proper transform of $C_{V, 0}:=$ $\mathbf{P}(V) \subset Y_{0}$ in $Y_{j}$ for $1 \leq j \leq i$. Let $E_{V, i+1}$ be the exceptional divisor of the blow-up along $C_{V, i}$ which is an irreducible component of $C_{i}$ and is identified with $\mathbf{P}(V)^{\mathcal{S}^{V}}$ (which is defined above). Let $E_{V, j}$ be the proper transform of $E_{V, i+1}$ in $Y_{j}$ for $i+1<j \leq n-2$. Finally, set $E_{V}=E_{V, n-2}$ if $\operatorname{dim} \mathbf{P}(V)<n-2$, and $E_{V}=C_{V, n-2}$ if $\operatorname{dim} \mathbf{P}(V)=n-2$. For $V=0$, let $E_{0}$ denote the proper transform $\widetilde{H}$ in $\widetilde{Y}$ of a general hyperplane $H$ of $\mathbf{P}^{n-1}$. (It is known that the divisor class group of $\widetilde{Y}$ is generated by $E_{V}$ for $V \in \mathcal{S}$ with $\operatorname{dim} \mathbf{P}(V)<n-2$.)

2.2. Remarks. (i) For smooth varieties $X \supset Y \supset Z$ in general, the proper transform of $Y$ by the blow-up of $X$ along $Z$ is the blow-up of $Y$ along $Z$.

(ii) For any linear subspaces $L, L^{\prime}$ of affine space such that $L \cap L^{\prime} \neq L, L^{\prime}$, the proper transforms of $L$ and $L^{\prime}$ by the blow-up along $L \cap L^{\prime}$ do not intersect.

2.3. Proposition. The union of $E_{V}$ for $V \in \mathcal{S} \backslash\{0\}$ is a divisor with normal crossings on $\tilde{Y}$, and the intersection of $E_{V_{k}}$ for $V_{k} \in \mathcal{S} \backslash\{0\}$ with $1 \leq k \leq r$ is empty unless $V_{1} \subset \cdots \subset V_{k}$ up to a permutation.

Proof. The last assertion follows from Remark (2.2)(i) because $\mathcal{S}$ is stable by intersection. For the first assertion we take local coordinates $x_{1}, \ldots, x_{n-1}$ such that $V_{k}$ is given by $x_{i}=0$ 
for $i>d_{k}$ where $d_{k}=\operatorname{dim} V_{k}$. Then local coordinates $y_{1}, \ldots, y_{n-1}$ of the blow-up along $V_{1}$ are given by $y_{i}=x_{i}$ if $i \leq d_{1}$ or $i=i_{1}$, and by $y_{i}=x_{i} / x_{i_{1}}$ otherwise. Here the exceptional divisor of the blow-up is given by $y_{i_{1}}=0$ and $i_{1}$ is an integer in $\left(d_{1}, d_{2}\right]$, because we have to consider the proper transforms of the $V_{k}$ for $k \geq 2$, which are given by $y_{i}=0$ for $i>d_{k}$ if $i_{1} \in\left(d_{1}, d_{2}\right]$. So the assertion follows by repeating this construction.

2.4. Proposition. The center $C_{i}$ of the blow-up $\rho_{i}$ is the disjoint union of $C_{V, i}=\mathbf{P}(V)^{\mathcal{S}^{V}}$ for $V \in \mathcal{S}$ with $\operatorname{dim} \mathbf{P}(V)=i$, and $C_{V, j+1}(1 \leq j<i)$ is the blow-up of $C_{V, j}$ along the disjoint union of $C_{V^{\prime}, j}$ for $V^{\prime} \in \mathcal{S}^{V}$ with $\operatorname{dim} \mathbf{P}\left(V^{\prime}\right)=j$.

Proof. This follows from Remarks (2.2)(i) and (ii) using the calculation in (2.3).

2.5. Proposition. Let $V, V^{\prime} \in \mathcal{S}$ such that $\operatorname{dim} \mathbf{P}(V)=i<\operatorname{dim} \mathbf{P}\left(V^{\prime}\right)=i^{\prime}$. Then $C_{V^{\prime}, i^{\prime}}$ is not contained in $E_{V, i^{\prime}}$, and hence $E_{V, j}$ inductively coincides with the total transform of $E_{V, i}$ for $j>i$.

Proof. This follows from the above arguments, because $\mathcal{S}$ is stable by intersections and the blow-ups are done by increasing induction on the dimension of the center. More precisely, we have $C_{V^{\prime}, i^{\prime}} \cap E_{V, i^{\prime}}=\emptyset$ in the case $V \not \subset V^{\prime}$. In the other case, repeating the above construction with $Y$ replaced by $\mathbf{P}\left(V^{\prime}\right)$, define $C_{V, i}^{\prime}, E_{V, i+1}^{\prime}$, and $E_{V}^{\prime}$ associated to $\mathbf{P}(V) \subset \mathbf{P}\left(V^{\prime}\right)$ as in (2.1) above, i.e. $E_{V, i+1}^{\prime}$ is the exceptional divisor of the blow-up along the center $C_{V, i}^{\prime}$, and $E_{V}^{\prime}$ is the proper transform of $E_{V, i+1}^{\prime}$ in $\mathbf{P}\left(V^{\prime}\right)^{\mathcal{S}^{\prime}}=C_{V^{\prime}, i^{\prime}}$, where the upper script ' of $C_{V, i}^{\prime}, E_{V, i+1}^{\prime}$ and $E_{V}^{\prime}$ means that the construction is done for $\mathbf{P}\left(V^{\prime}\right)$ instead of $Y$. Then $C_{V^{\prime}, i^{\prime}} \cap E_{V, i^{\prime}}=E_{V}^{\prime}$. This finishes the proof of Proposition (2.5).

2.6. Proposition. For $V \in \mathcal{S}, E_{V}$ depends only on $\mathcal{S}^{V}$ and $\mathcal{S}_{V}$, and there is a canonical decomposition

$$
E_{V}=\mathbf{P}(V)^{\mathcal{S}^{V}} \times \mathbf{P}(X / V)^{\mathcal{S}_{V}}
$$

where $\mathbf{P}(V)^{\mathcal{S}^{V}}, \mathbf{P}(X / V)^{\mathcal{S}_{V}}$ are the successive blow-ups of $\mathbf{P}(V)$ and $\mathbf{P}(X / V)$ respectively associated with $\mathcal{S}^{V}$ and $\mathcal{S}_{V}$ as in $(2.1)$.

Proof. The first assertion is clear because $\mathcal{S}$ is stable by intersections. Let $r$ be the codimension of $\mathbf{P}(V)$ in $Y$ (i.e. $r=n-1-i$ ). Taking $r$ general hyperplanes containing $V$, and considering their proper transforms whose intersection is $C_{V, i}$, we see that the tensor of the conormal bundle of $C_{V, i}$ with some line bundle is a trivial vector bundle. Hence $E_{V, i+1}$ is a trivial $\mathbf{P}^{r-1}$-bundle over $C_{V, i}$ with a canonical projection to $\mathbf{P}^{r-1}$ (which is independent of the choice of the hyperplanes up to the action of $P G L(r-1, \mathbf{C}))$. So the assertion follows, because $\mathcal{S}_{V}$ is identified with a set of vector subspaces of $X / V$.

2.7. Proposition. Let $V_{k} \in \mathcal{S}$ for $1 \leq k \leq r$ such that $0 \neq V_{1} \varsubsetneqq \cdots \varsubsetneqq V_{r}$. Then

$$
\bigcap_{1 \leq k \leq r} E_{V_{k}}=\prod_{0 \leq k \leq r} \mathbf{P}\left(V_{k+1} / V_{k}\right)^{\mathcal{S}_{k}},
$$

where $V_{0}=0, V_{r+1}=X$, and $\mathcal{S}_{k}=\left\{V / V_{k} \mid V \in \mathcal{S}\right.$ with $\left.V_{k} \subset V \varsubsetneqq V_{k+1}\right\}$.

Proof. Let $V=V_{r}$ and $i=\operatorname{dim} \mathbf{P}(V)$. Then $E_{V} \cap E_{V_{k}}$ is the pull-back of $C_{V, i} \cap E_{V_{k}, i}$ by the projection $E_{V} \rightarrow C_{V, i}$ for $1 \leq k \leq r-1$. So the assertion follows from (2.6) 
by induction on $r$, where the inductive hypothesis is applied to $C_{V, i}=\mathbf{P}(V)^{\mathcal{S}^{V}}$ and $C_{V, i} \cap E_{V_{k}, i}(1 \leq k \leq r-1)$ which are calculated in the proof of Proposition (2.5).

2.8. Proposition. For $V^{\prime} \in \mathcal{S}^{V}$ (resp. $\left.\mathcal{S}_{V}\right)$ such that $V^{\prime} \neq V$, the pull-back of $E_{V^{\prime}}$ to $E_{V}$ coincides with the pull-back of $E_{V^{\prime}}^{\prime}$ on $\mathbf{P}(V)^{\mathcal{S}^{V}}$ by $r_{1}\left(\right.$ resp. of $E_{V^{\prime}}^{\prime \prime}$ on $\mathbf{P}(X / V)^{\mathcal{S}_{V}}$ by $\left.p_{2}\right)$. Here $\mathbf{P}(V)^{\mathcal{S}^{V}}$ and $\mathbf{P}(X / V)^{\mathcal{S}_{V}}$ are as in (2.6), and $\mathrm{pr}_{i}$ denote the projection to the $i$-th factor. For $V^{\prime}=V$, the pull-back of $E_{V}$ to $E_{V}$ as a divisor class is given by

$$
p r_{1}^{*}\left(\widetilde{H}^{\prime}-\sum_{V^{\prime} \in \mathcal{S}^{V}} E_{V^{\prime}}^{\prime}\right)-p r_{2}^{*} \widetilde{H}^{\prime \prime}
$$

where $\widetilde{H}^{\prime}$ (resp. $\left.\widetilde{H}^{\prime \prime}\right)$ is the proper transform of a general hyperplane of $\mathbf{P}(V)$ (resp. $\mathbf{P}(X / V)$ ), and it is zero if $\operatorname{dim} \mathbf{P}(V)=0$ (resp. $\operatorname{dim} \mathbf{P}(X / V)=0)$.

Proof. The first assertion follows from (2.5-6). For the second, take a general hyperplane of $Y$ intersecting $\mathbf{P}(V)$ transversally and also a hyperplane of $Y$ containing $\mathbf{P}(V)$ and corresponding to a general hyperplane of $\mathbf{P}(X / V)$. Then the assertion follows by considering the difference between their pull-backs to $\widetilde{Y}$.

\section{Proofs of Theorems 3 and 5}

3.1. Proof of Theorem 3. This follows from Proposition (1.5) together with the Riemann-Roch theorem for surfaces (see e.g. [17], Example 15.2.2)

$$
\chi\left(\mathcal{O}_{\widetilde{Y}}\left(D_{k}\right)\right)=\frac{1}{2} D_{k} \cdot\left(D_{k}-K_{\widetilde{Y}}\right)+\chi\left(\mathcal{O}_{\widetilde{Y}}\right)
$$

Here $\tilde{Y}$ is the blow-up of $Y=\mathbf{P}^{2}$ along the points of $\mathbf{P}\left(D^{\text {nnc }}\right)$ in the notation of $(0.1)$, and $D_{k}$ is a divisor on it (which will be defined later).

By (1.4.1) we may assume

$$
\alpha=\frac{i}{d}+\ell \quad \text { with } i=d-k \in[1, d], \ell=2-p \in[0,2]
$$

since $n_{f, \alpha}=0$ for the other $\alpha$. Consider first the case $\ell=0$. We have

$$
\left.\Omega_{\widetilde{Y}}^{2}(\log \widetilde{Z})=\mathcal{O}_{\widetilde{Y}}\left((d-3) \widetilde{H}+\sum_{j \in J^{\prime \prime}}\left(2-m_{j}\right) E_{j}\right)\right),
$$

since $\Omega_{\widetilde{Y}}^{2}=\rho^{*} \Omega_{Y}^{2} \otimes_{\mathcal{O}} \mathcal{O}_{\widetilde{Y}}\left(\sum_{j \in J^{\prime \prime}} E_{j}\right)$ and $\widetilde{Z}_{\text {red }}=\rho^{*} Z+\sum_{j \in J^{\prime \prime}}\left(1-m_{j}\right) E_{j}$ in the notation of (1.4). So we apply the Riemann-Roch theorem (3.1.1) to

$$
D_{k}=(d-k-3) \widetilde{H}+\sum_{j \in J^{\prime \prime}}\left(-m_{j}+\left\lfloor k m_{j} / d\right\rfloor+2\right) E_{j}
$$

using Proposition (1.5) for $p=2$. We have $D_{k}=\sum_{j} A_{j} E_{j}+C \widetilde{H}$ with

$$
A_{j}=2+\left\lfloor-i m_{j} / d\right\rfloor=2-\left\lceil i m_{j} / d\right\rceil, \quad C=i-3,
$$


and $K_{\widetilde{Y}}=-3 \widetilde{H}+\sum_{j} E_{j}$. So we get

$$
D_{k}^{2}=-\sum_{j} A_{j}^{2}+C^{2}, \quad D_{k} \cdot K_{\widetilde{Y}}=-\sum_{j} A_{j}-3 C
$$

since $\widetilde{H}^{2}=1, E_{j}^{2}=-1$, and $\widetilde{H}, E_{j}$ are orthogonal to each other. These imply the first equality by $(3.1 .1)$ since $\chi\left(\mathcal{O}_{\widetilde{Y}}\right)=1$. with

The argument is similar for the last equality where $\ell=2, p=0, D_{k}=\sum_{j} A_{j} E_{j}+C \widetilde{H}$

$$
A_{j}=\left\lfloor k m_{j} / d\right\rfloor=m_{j}-\left\lceil i m_{j} / d\right\rceil, \quad C=-k=i-d .
$$

Note that the reduced cohomology is used for the definition of spectrum, and the difference corresponds to $\delta_{i, d}$ in the case $i=d$.

By the identity $\left(\begin{array}{c}a+b \\ 2\end{array}\right)-\left(\begin{array}{l}a \\ 2\end{array}\right)-\left(\begin{array}{l}b \\ 2\end{array}\right)=a b$, the middle equality for $\ell=1$ follows from the others since we have by (1.4.2)

$$
\sum_{\ell=0}^{2} n_{f, \frac{i}{d}+\ell}=\chi(U)-\delta_{i, d} \quad \text { with } \quad \chi(U)=\left(\begin{array}{c}
d-2 \\
2
\end{array}\right)-\sum_{m \geq 3} \nu_{m}^{(2)}\left(\begin{array}{c}
m-1 \\
2
\end{array}\right) .
$$

Here the first equality is clear by the definition of spectrum. The last equality is shown by using a small deformation to a generic central arrangement $D^{\prime}$ where $\mathbf{P}\left(D^{\prime}\right)$ is a divisor with normal crossing so that

$$
\chi\left(\mathbf{P}^{2} \backslash \mathbf{P}\left(D^{\prime}\right)\right)=3-2 d-\left(\begin{array}{l}
d \\
2
\end{array}\right)=\left(\begin{array}{c}
d-2 \\
2
\end{array}\right) .
$$

The difference of the local Euler characteristics of $\mathbf{P}(D)$ and $\mathbf{P}\left(D^{\prime}\right)$ at each point of $\mathbf{P}(D)$ with multiplicity $m$ is given by $1-\left(m-\left(\begin{array}{c}m \\ 2\end{array}\right)\right)=\left(\begin{array}{c}m-1 \\ 2\end{array}\right)$. So the assertion follows.

3.2. Generic case. Assume $D$ is a generic central hyperplane arrangement, i.e. $\mathbf{P}(D) \subset$ $\mathbf{P}^{n-1}$ is a divisor with normal crossings. In this case it is known ([27], Cor. 1) that

$$
n_{f, \alpha}=n_{f, n-\alpha}=\left(\begin{array}{c}
i-1 \\
n-1
\end{array}\right) \text { for } \alpha=i / d<1,
$$

where $n_{f, \alpha}=0$ for $d \alpha \notin \mathbf{Z}$. For $\alpha=i \in \mathbf{Z}$, we have by 5.6.1 in loc. cit.

$$
n_{f, i}=(-1)^{i-1}\left(\begin{array}{l}
d-1 \\
n-i
\end{array}\right) \quad \text { for } 1 \leq i \leq n-1 .
$$

It is possible to calculate $n_{f, \alpha}$ for any $\alpha$ using Proposition (1.5) and the Bott vanishing theorem.

3.3. Mustață's formula. In the notation of (1.1), set $\mathcal{S}^{\prime}=\mathcal{S}\left(D^{\text {nrnc }}\right)$. For each $V \in \mathcal{S}^{\prime}$, let $\mathcal{I}_{V} \subset \mathbf{C}[X]$ be the reduced ideal of $V$. Mustaţă's formula [22] states that for any $\alpha>0$

$$
\mathcal{J}(X, \alpha D)=\bigcap_{V \in \mathcal{S}^{\prime}} \mathcal{I}_{V}^{\lfloor\alpha \mu(V)\rfloor-\gamma(V)+1} .
$$

In the nonreduced case this is due to Z. Teitler [32] (see also [27], 2.2).

If $V=0$, then we have for $\alpha=j / d$ with $j \in[1, d] \cap \mathbf{Z}$

$$
\mathcal{I}_{0}^{\lfloor(\alpha-\varepsilon) \mu(0)\rfloor-(\gamma(0))+1}=\mathcal{I}_{0}^{j-n},
$$


for $0<\varepsilon \ll 1 / d$, since $\gamma(0)=n$ and $\mu(0)=\operatorname{deg} D=d$. So we get $(0.1)$ where $n=3$, see also [22], Cor. 2.1.

3.4. Proof of Theorem 5. By (1.4.3-5) for $p=n-1$, we get for $\alpha=\frac{i}{d} \in(0,1]$ with $i=d-k \in[1, d]$

$$
\begin{aligned}
n_{f, \alpha} & =\operatorname{dim} \Gamma\left(\widetilde{Y}, \mathcal{O}_{\widetilde{Y}}\left((i-n) \widetilde{H}+\sum_{j \in J}\left(c_{j}-\left\lceil i m_{j} / d\right\rceil\right) E_{j}\right)\right) \\
& =\operatorname{dim}\left(\bigcap_{V \in \mathcal{S}^{\prime} \backslash\{0\}} \mathcal{I}_{V}^{\lceil\alpha \mu(V)\rceil-\gamma(V)} \cap \mathbf{C}[X]_{i-n}\right),
\end{aligned}
$$

where the second equality is shown by using the injection

$$
\begin{aligned}
& \Gamma\left(\widetilde{Y}, \mathcal{O}_{\widetilde{Y}}\left((i-n) \widetilde{H}+\sum_{j}\left(c_{j}-\left\lceil i m_{j} / d\right\rceil\right) E_{j}\right)\right. \\
& \subset \Gamma\left(\widetilde{Y}, \mathcal{O}_{\widetilde{Y}}((i-n) \widetilde{H})\right)=\Gamma\left(Y, \mathcal{O}_{Y}(i-n)\right)=\mathbf{C}[X]_{i-n}
\end{aligned}
$$

Indeed, for $g \in \mathbf{C}[X]_{i-n}=\Gamma\left(\widetilde{Y}, \mathcal{O}_{\widetilde{Y}}((i-n) \widetilde{H})\right)$, the condition $g \in \mathcal{I}_{V}^{k}$ corresponds to that $\pi^{*} g \in \mathcal{I}_{E_{j}}^{k}$ if $E_{j}$ corresponds to $V$, where $\mathcal{I}_{E_{j}}$ is the ideal of $E_{j}$ and

$$
k=\left\lceil i m_{j} / d\right\rceil-c_{j}=\lceil\alpha \mu(V)\rceil-\gamma(V) .
$$

Note that we may have $c_{j}-\left\lceil i m_{j} / d\right\rceil>0$ only in the case $c_{j} \geq 2$ so that $g$ cannot have a pole. Thus the assertion follows.

3.5. Relation with $b$-functions. It does not seem easy to get an explicit formula for the jumping coefficients and the spectrum of a hyperplane arrangement in the general case. However, it seems to be more difficult to calculate the roots of the $b$-function of a hyperplane arrangement except for the case of a generic central arrangement [33], see also [27]. The relation between the jumping coefficients $\mathrm{JC}(D)$ and the roots of the $b$-function $R_{f}$ is quite complicated although there is an inclusion relation

$$
\mathrm{JC}(D) \cap(0,1) \subset R_{f} \cap(0,1)
$$

as is shown in [14]. The converse inclusion holds under some conditions, see [27]. However, it does not hold without these conditions as is shown by the following.

3.6. Example. Let $n=3, d=7$ and $f=\left(x^{2}-y^{2}\right)\left(x^{2}-z^{2}\right)\left(y^{2}-z^{2}\right) z$. By (0.1) or Theorem 3 we have $\frac{5}{7} \notin \mathrm{JC}(D)$, but $\frac{5}{7} \in R_{f}$, see [28]. In this case

$$
\operatorname{dim} \mathbf{C}[x, y, z]_{2}=\# \mathbf{P}\left(D^{\mathrm{nnc}}\right)=6
$$

So we have to prove the non-degeneracy of some matrix to show the non-existence of a nontrivial homogeneous polynomial of degree 2 vanishing at all the 6 points if we want to show that $\frac{5}{7} \notin \mathrm{JC}(D)$ using (0.1). Note that $n_{f, 5 / 7}=0$ by Theorem 3 where $d=7$, $\nu_{3}^{(2)}=6$ and $\nu_{m}^{(2)}=0(m>3)$. This implies that $\frac{5}{7} \notin \mathrm{JC}(D)$ by Proposition (4.2). 


\section{Proofs of Theorems $1-4$ by induction}

4.1. Isolated jumping coefficients. Let $X$ be a smooth variety (or a complex manifold), and $D$ be a divisor on it. Let $\mathcal{J}(X, \alpha D)$ denote the multiplier ideals for $\alpha>0$, see [21]. The graded pieces of the multiplier ideals are defined for $\alpha>0$ with $0<\varepsilon \ll 1$ by

$$
\mathcal{G}(X, \alpha D)=\mathcal{J}(X,(\alpha-\varepsilon) D) / \mathcal{J}(X, \alpha D) .
$$

The jumping coefficients are the rational numbers $\alpha$ such that $\mathcal{G}(X, \alpha D) \neq 0$. We say that $\alpha$ is an isolated jumping coefficient at $x$ if $\mathcal{G}(X, \alpha D)$ is supported on $x$.

If $D$ has an isolated singularity at $x$ and is defined locally by $f$, then the coefficient $n_{f, \alpha}$ of the spectrum $\operatorname{Sp}(f)=\sum_{\alpha>0} n_{f, \alpha} t^{\alpha}$ for $\alpha \in(0,1)$ is given (see [3]) by

$$
n_{f, \alpha}=\operatorname{dim} \mathcal{G}(X, \alpha D)_{x} .
$$

4.2. Proposition. The assertion (4.1.1) holds by assuming only that $\alpha \in(0,1)$ is an isolated jumping coefficient at $x$.

Proof. By [6], we have a canonical isomorphism

$$
\mathcal{G}(X, \alpha D)=F_{-n} \operatorname{Gr}_{V}^{\alpha} \mathcal{B}_{f}
$$

where $\operatorname{Gr}_{V}^{\alpha} \mathcal{B}_{f}$ coincides with the $\lambda$-eigenspace of $\psi_{f} \mathcal{O}_{X}$ by the action of the monodromy where $\lambda=e^{-2 \pi i \alpha}$. Here we have to show that $F_{-n}$ does not change by taking the pull-back by $i_{x}:\{x\} \rightarrow X$ as in (1.6). Choosing local coordinates $x_{1}, \ldots, x_{n}$ of $(X, x)$ and using [26], 2.24, the pull-back $i_{x}^{*}$ is given by iterating

$$
i_{k}^{*}=C\left(\operatorname{can}: \psi_{x_{k}, 1} \rightarrow \varphi_{x_{k}, 1}\right) .
$$

For the underlying filtered left $D$-modules $(M, F)$, the last functor is given by the mapping cone of

$$
\partial_{x_{k}}: \operatorname{Gr}_{V_{k}}^{1}(M, F[1]) \rightarrow \operatorname{Gr}_{V_{k}}^{0}(M, F),
$$

where $V_{k}$ is the $V$-filtration along $x_{k}=0$ and $x_{k} \partial_{k}-\alpha$ is nilpotent on $\operatorname{Gr}_{V_{k}}^{\alpha}$. Since $\operatorname{supp} F_{-n} M=\{x\}$, we see that $F_{-n} M$ is contained in

$$
\left(i_{x}\right)_{*} H^{0} i_{x}^{!} M=\Gamma_{[x]} M \subset M,
$$

which underlies a mixed Hodge module, and is isomorphic to $\bigoplus_{i}\left(\mathbf{C}\left[\partial_{1}, \ldots, \partial_{n}\right], F\left[p_{i}\right]\right)$ with $p_{i} \in \mathbf{Z}$. (Indeed, it is the direct image as a $D$-module of the filtered vector space $H^{0} i_{x}^{!}(M, F)$ by the closed embedding $i_{x}$. Note that $\mathbf{C}\left[\partial_{1}, \ldots, \partial_{n}\right]$ has the Hodge filtration $F$ by the degree of polynomials in $\partial_{i}$.) So $F_{-n} M$ does not change by passing to $\operatorname{Gr}_{V_{k}}^{0}(M, F)$ inductively. Since $F_{-n}=0$ on $(M, F[1])$, this implies the desired result.

4.3. Proof of Proposition 1. Assume $\alpha \in \operatorname{JC}(D) \cap(0,1)$. It is well-known that $\mathcal{G}(X, \alpha D)=0$ for $\alpha \in(0,1)$ if $D$ is a reduced divisor with normal crossings. So the 
support of $\mathcal{G}(X, \alpha D)$ is a union of $V \in \mathcal{S}(D)^{\text {nrnc }}$, since $D$ is locally trivial along a nonempty Zariski open subset of $V$. Restricting $D$ to an affine subspace which is transversal to the Zariski open subset of $V \in \mathcal{S}(D)^{\text {nrnc }}$ and has complementary dimension with $V$, it is enough to consider the case of isolated jumping coefficients, since $D$ is locally the product of its restriction to the transversal space with $V$. (But this does not mean that the singular points of $D$ are 0-dimensional.) Then we get the non-vanishing of $n_{f_{X / V}, \alpha}$ by Proposition (4.2) since $f_{X / V}$ is identified with the defining polynomial of the restriction of $D$ to the transversal space. So Proposition 1 follows since the opposite implication is well-known, see [3].

4.4. Theorem. In the notation of (1.1) and (2.1), set $\mathcal{S}=\mathcal{S}(D)^{\mathrm{nnc}}$ and $\mathcal{S}^{D_{i}}=\{V \in \mathcal{S} \mid$ $\left.V \subset D_{i}\right\}$. Let $\widetilde{Z}$ be as in (1.4). For $a=\left(a_{V}\right)_{V \in \mathcal{S}} \in \mathbf{Z}^{\mathcal{S}}$ define

$$
\Phi_{\mathcal{S}}^{p}(a)=\chi\left(\widetilde{Y}, \Omega_{\widetilde{Y}}^{p}(\log \widetilde{Z}) \otimes_{\mathcal{O}} \mathcal{O}_{\widetilde{Y}}\left(\sum_{V \in \mathcal{S}} a_{V} E_{V}\right)\right)
$$

Then $\Phi_{\mathcal{S}}^{p}(a)$ is a polynomial in $a=\left(a_{V}\right)_{V \in \mathcal{S}}$ whose coefficients are rational numbers and are determined by the combinatorial data of D. More precisely, it depends only on $\mathcal{S}, \subset, \mu_{\mathrm{red}}, \gamma$ together with $\mathcal{S}^{D_{i}}(i \in \Lambda)$ in the notation of (1.1.1). If $p=0$, then $\Phi_{\mathcal{S}}^{0}(a)$ depends only on the weak combinatorial data.

Proof. We show this by increasing induction on $n=\operatorname{dim} X \geq 2$. First we show the assertion on $\Phi_{\mathcal{S}}^{p}(a)$ for any $p$. If $n=2$, the assertion is trivial by the Riemann-Roch theorem for curves. Here $\mathcal{S}=\{0\}$ and the number of the points of $\widetilde{Z}$ is enough for the calculation.

Assume $n>2$, and set

$$
M(a)=\Omega_{\widetilde{Y}}^{p}(\log \widetilde{Z}) \otimes_{\mathcal{O}} \mathcal{O}_{\widetilde{Y}}\left(\sum_{V \in \mathcal{S}} a_{V} E_{V}\right)
$$

Here we may assume $p<\operatorname{dim} \tilde{Y}$ since the case $p=\operatorname{dim} \tilde{Y}$ is reduced to the case $p=0$. Take some $V \in \mathcal{S}$, and set $E=E_{V}$ if $V \neq 0$. In the case $V=0$, set $E=\widetilde{H}$ which is the pull-back of a sufficiently general hyperplane. We have a short exact sequence

$$
0 \rightarrow M\left(a-\mathbf{1}_{V}\right) \rightarrow M(a) \rightarrow M(a) \otimes_{\mathcal{O}_{\widetilde{Y}}} \mathcal{O}_{E} \rightarrow 0
$$

where $\mathbf{1}_{V} \in \mathbf{Z}^{\mathcal{S}}$ is defined by $\left(\mathbf{1}_{V}\right)_{V^{\prime}}=0$ for $V^{\prime} \neq V$ and $\left(\mathbf{1}_{V}\right)_{V}=1$. So we get

$$
\Phi_{\mathcal{S}}^{p}(a)-\Phi_{\mathcal{S}}^{p}\left(a-\mathbf{1}_{V}\right)=\chi\left(E, M(a) \otimes_{\mathcal{O}_{\widetilde{Y}}} \mathcal{O}_{E}\right)
$$

Using the identity $\left(\begin{array}{l}x \\ k\end{array}\right)-\left(\begin{array}{c}x-1 \\ k\end{array}\right)=\left(\begin{array}{l}x-1 \\ k-1\end{array}\right)$ as polynomials in $x$ where $k \in \mathbf{Z}_{>0}$ (see also [18], I, Prop. 7.3(a)), it is enough to show that (4.4.1) is a polynomial determined by the combinatorial data.

We consider first the case $V \neq 0$. Let $N_{E}^{*}$ denote the conormal bundle of $E \subset \tilde{Y}$. This is the restriction of the line bundle $\mathcal{O}_{\widetilde{Y}}(-E)$, and the restriction as a divisor is calculated 
in (2.8). Let $\widetilde{Z}^{\prime}$ be the closure of $\widetilde{Z} \backslash E$. Set $\widetilde{Z}_{E}^{\prime}=\widetilde{Z}^{\prime} \cap E$. There is a commutative diagram of exact sequences

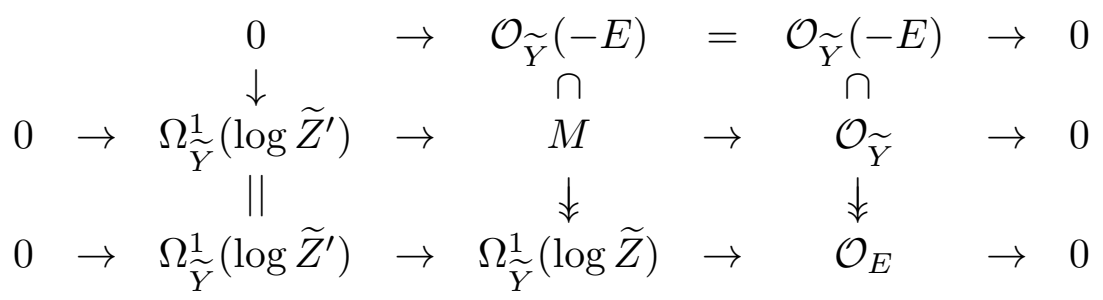

where $M:=\operatorname{Ker}\left(\Omega_{\widetilde{Y}}^{1}(\log \widetilde{Z}) \oplus \mathcal{O}_{\widetilde{Y}} \rightarrow \mathcal{O}_{E}\right)$. Taking the pull-back by $E \rightarrow \widetilde{Y}$, we get short exact sequences

$$
\begin{aligned}
0 & \left.\left.\rightarrow N_{E}^{*} \rightarrow M\right|_{E} \rightarrow \Omega_{\widetilde{Y}}^{1}(\log \widetilde{Z})\right|_{E} \rightarrow 0, \\
\left.0 \rightarrow \Omega_{\widetilde{Y}}^{1}\left(\log \widetilde{Z}^{\prime}\right)\right|_{E} & \left.\rightarrow M\right|_{E} \rightarrow \mathcal{O}_{E} \rightarrow 0 .
\end{aligned}
$$

We have also a short exact sequence

$$
\left.0 \rightarrow N_{E}^{*} \rightarrow \Omega_{\widetilde{Y}}^{1}\left(\log \widetilde{Z}^{\prime}\right)\right|_{E} \rightarrow \Omega_{E}^{1}\left(\log \left(\widetilde{Z}_{E}^{\prime}\right) \rightarrow 0 .\right.
$$

These imply the equalities in the Grothendieck group

$$
\begin{aligned}
{\left[\left.\bigwedge^{p} M\right|_{E}\right]=} & {\left[\left.\Omega_{\widetilde{Y}}^{p}(\log \widetilde{Z})\right|_{E}\right]+\left[\left.N_{E}^{*} \otimes \Omega_{\widetilde{Y}}^{p-1}(\log \widetilde{Z})\right|_{E}\right] } \\
{\left[\left.\bigwedge^{p} M\right|_{E}\right]=} & {\left[\left.\Omega_{\widetilde{Y}}^{p}\left(\log \widetilde{Z}^{\prime}\right)\right|_{E}\right]+\left[\left.\Omega_{\widetilde{Y}}^{p-1}\left(\log \widetilde{Z}^{\prime}\right)\right|_{E}\right] } \\
= & {\left[\Omega_{E}^{p}\left(\log \widetilde{Z}_{E}^{\prime}\right)\right]+\left[N_{E}^{*} \otimes \Omega_{E}^{p-1}\left(\log \widetilde{Z}_{E}^{\prime}\right)\right] } \\
& +\left[\Omega_{E}^{p-1}\left(\log \widetilde{Z}_{E}^{\prime}\right)\right]+\left[N_{E}^{*} \otimes \Omega_{E}^{p-2}\left(\log \widetilde{Z}_{E}^{\prime}\right)\right]
\end{aligned}
$$

So we get by increasing induction on $p$

$$
\left[\left.\Omega_{\widetilde{Y}}^{p}(\log \widetilde{Z})\right|_{E}\right]=\left[\Omega_{E}^{p}\left(\log \widetilde{Z}_{E}^{\prime}\right)\right]+\left[\Omega_{E}^{p-1}\left(\log \widetilde{Z}_{E}^{\prime}\right)\right]
$$

The assertion on (4.4.1) is thus reduced to that

$$
\chi\left(E,\left.\Omega_{E}^{p}\left(\log \widetilde{Z}_{E}^{\prime}\right) \otimes_{\mathcal{O}} \mathcal{O}_{\widetilde{Y}}\left(\sum_{V \in \mathcal{S}} a_{V} E_{V}\right)\right|_{E}\right)
$$

is a polynomial determined by the combinatorial data. By Proposition (2.6) we have

$$
E=\mathbf{P}(V)^{\mathcal{S}^{V}} \times \mathbf{P}(X / V)^{\mathcal{S}_{V}}
$$

and the restriction of $\left.\mathcal{O}_{\widetilde{Y}}\left(\sum_{V \in \mathcal{S}} a_{V} E_{V}\right)\right)$ to $E$ is calculated by Proposition (2.8). Moreover we have the decomposition

$$
\widetilde{Z}_{E}^{\prime}=p r_{1}^{*} Z_{1}+p r_{2}^{*} Z_{2}
$$

where $Z_{1}$ is given by $E_{V^{\prime}}^{\prime}$ with $V^{\prime} \subset V$, and $Z_{2}$ is given by $E_{D_{i} / V}^{\prime \prime}, E_{V^{\prime} / V}^{\prime \prime}$ with $D_{i}, V^{\prime} \supset$ $V$. Here $E_{D_{i} / V}^{\prime \prime} \subset \mathbf{P}(X / V)^{\mathcal{S}_{V}}$ is the proper transform of $\mathbf{P}\left(D_{i} / V\right) \subset \mathbf{P}(X / V)$, and the 
associated combinatorial data $\left(\mathcal{S}_{V}\right)^{D_{i} / V}$ is given by $\mathcal{S}_{V} \cap \mathcal{S}^{D_{i}}$. So the assertion after (4.4.1) for $V \neq 0$ follows from the inductive assumption using the Künneth-type decomposition of $\Omega_{E}^{p}\left(\log \widetilde{Z}_{E}^{\prime}\right)$.

For $V=0$ we have a similar assertion since $\widetilde{H}$ intersects transversally $E_{V}$ for every $V \in \mathcal{S} \backslash\{0\}$. Using an exact sequence similar to (4.4.2), we get instead of (4.4.3)

$$
\left[\left.\Omega_{\widetilde{Y}}^{p}(\log \widetilde{Z})\right|_{\widetilde{H}}\right]=\left[\Omega_{\widetilde{H}}^{p}\left(\log \widetilde{Z}_{\widetilde{H}}\right)\right]+\left[N_{\widetilde{H}}^{*} \otimes \Omega_{\widetilde{H}}^{p-1}\left(\log \widetilde{Z}_{\widetilde{H}}\right)\right]
$$

Applying the inductive hypothesis, we can then calculate

$$
\chi\left(\widetilde{H},\left.\left.\Omega_{\widetilde{Y}}^{p}(\log \widetilde{Z})\right|_{\widetilde{H}} \otimes_{\mathcal{O}} \mathcal{O}_{\widetilde{Y}}\left(\sum_{V \in \mathcal{S}} a_{V} E_{V}\right)\right|_{\widetilde{H}}\right),
$$

where $\mathcal{S}^{D_{i}}$ and $\mathcal{S}$ are replaced by those obtained by deleting the 1-dimensional $V$ (i.e. $\operatorname{dim} \mathbf{P}(V)=0)$. So the assertion after (4.4.1) for $V=0$ is also proved.

Thus the assertion is reduced to the case $a=0$, and we have to show that

$$
\Phi_{\mathcal{S}}^{p}(0)=\chi\left(\widetilde{Y}, \Omega_{\widetilde{Y}}^{p}(\log \widetilde{Z})\right)
$$

depends only on the combinatorial data. It is known that each $H^{j}(U)$ is generated by products of logarithmic 1-forms (see [2]), and hence has type $(j, j)$. Then the assertion that the Hodge numbers of $U=\widetilde{Y} \backslash \widetilde{Z}$ depend only on the combinatorial data is equivalent to a similar assertion for the Betti numbers. So the assertion follows from Proposition (1.10).

The argument is similar and easier for the assertion on the weak combinatorial data in the case $p=0$, since we do not have to treat the logarithmic forms by the isomorphism $\Omega_{\widetilde{Y}}^{0}(\log \widetilde{Z})=\mathcal{O}_{\widetilde{Y}}$. This finishes the proof of Theorem (4.4).

4.5. Proofs of Theorems 1-2 by induction. Theorem 1 follows from Theorem (4.4) and Proposition (1.5). If $D$ is reduced, then $\left\lfloor k m_{j} / d\right\rfloor=0$ for $j \in J^{\prime}$ in the notation of (1.4), and $c_{j}-m_{j}=0$ for $j \in J^{\prime}$ in (1.4.5). Using the second equality of (1.4.5) for $p=n-1$, Theorem 2 then follows from Theorem (4.4) and Proposition (1.5).

4.6. Remark. In the original version [29], the argument in the proof of Theorem (4.4) treated $\Omega_{\widetilde{Y}}^{p}$ and not $\Omega_{\widetilde{Y}}^{p}(\log \widetilde{Z})$. By this method we have to take the graded pieces of the weight filtration $W$ on the logarithmic forms, and the argument becomes more complicated. The above proof of Theorem 1 was obtained after the new proof in the next section appeared.

We can calculate examples using the method in this section as is shown below.

4.7. Proof of Theorem 3 by induction. Let $\Phi^{p}(A, C)$ denote $\Phi_{\mathcal{S}}^{p}(a)$ in (4.4) where $A=\left(A_{j}\right)$, and the $a_{V}$ are denoted by $A_{j}$ or $C$ depending on whether $\operatorname{dim} V=1$ or 0 . Let $E_{j}$ denote the exceptional divisor corresponding to $A_{j}$. Then

$$
\Phi(A, C)=\chi(\widetilde{Y}, \mathcal{E}) \quad \text { with } \quad \mathcal{E}=\mathcal{O}_{\widetilde{Y}}\left(\sum_{j} A_{j} E_{j}+C \widetilde{H}\right)
$$

We have $\mathbf{R} \pi_{*} \mathcal{O}_{\widetilde{Y}}=\mathcal{O}_{Y}$ where $\pi: \tilde{Y} \rightarrow Y$ since $Y$ is nonsingular. So we first get

$$
\Phi^{0}(0, C)=\left(\begin{array}{c}
C+2 \\
2
\end{array}\right)
$$


Fix $j$, and let $\mathbf{1}_{j}$ be as in the proof of Theorem (4.4). We have $\left.\mathcal{O}_{\widetilde{Y}}\left(E_{j}\right)\right|_{E_{j}}=\mathcal{O}_{E_{j}}(-1)$ where $E_{j}=\mathbf{P}^{1}$. (This is shown by using the total transform of a hyperplane passing through the center of the blow-up.) Hence

$$
\Phi^{0}(A, C)-\Phi^{0}\left(A-\mathbf{1}_{j}, C\right)=\chi\left(E_{j}, \mathcal{O}_{E_{j}}\left(-A_{j}\right)\right)=1-A_{j} .
$$

Thus we get

$$
\Phi^{0}(A, C)=\left(\begin{array}{c}
C+2 \\
2
\end{array}\right)-\sum_{j}\left(\begin{array}{c}
A_{j} \\
2
\end{array}\right) .
$$

This implies the assertions for $\alpha \in(0,1] \cup(2,3]$ by setting $A_{j}, C$ as in (3.1).

For $p=1$, we have by (4.4.4) applied to a general $H=\mathbf{P}^{1} \subset Y$

$$
\begin{aligned}
\Phi^{1}(0, C)-\Phi^{1}(0, C-1) & =\chi\left(\mathbf{P}^{1}, \Omega_{\mathbf{P}^{1}}^{1} \otimes \mathcal{O}_{\mathbf{P}^{1}}(C+d)\right)+\chi\left(\mathbf{P}^{1}, N_{H}^{*} \otimes \mathcal{O}_{\mathbf{P}^{1}}(C)\right) \\
& =(C+d-1)+C=2 C+d-1 .
\end{aligned}
$$

Since $\Phi^{1}(0,0)=b_{1}(U)=d-1$, we get

$$
\Phi^{1}(0, C)=C^{2}+d C+d-1 .
$$

Fix now $j$. We have by (4.4.3)

$$
\begin{aligned}
\Phi^{1}(A, C)-\Phi^{1}\left(A-\mathbf{1}_{j}, C\right) & =\chi\left(\mathbf{P}^{1}, \Omega_{\mathbf{P}^{1}}^{1} \otimes \mathcal{O}_{\mathbf{P}^{1}}\left(m_{j}-A_{j}\right)\right)+\chi\left(\mathbf{P}^{1}, \mathcal{O}_{\mathbf{P}^{1}}\left(-A_{j}\right)\right) \\
& =\left(m_{j}-A_{j}-1\right)+\left(1-A_{j}\right)=m_{j}-2 A_{j} .
\end{aligned}
$$

We get thus

$$
\Phi^{1}(A, C)=\sum_{j}\left(-A_{j}^{2}-A_{j}+m_{j} A_{j}\right)+C^{2}+d C+d-1 .
$$

Here $A_{j}=m_{j}-\left\lceil i m_{j} / d\right\rceil, C=i-d$ by Proposition (1.5). So the assertion follows.

4.8. Proof of Theorem 4 by induction. Let $\Phi(A, B, C)$ denote $\Phi_{\mathcal{S}}^{0}(a)$ in (4.4) where $A=\left(A_{j}\right), B=\left(B_{k}\right)$, and $A_{j}, B_{k}, C$ denote $a_{V}$ depending on whether $\operatorname{dim} V=2,1,0$. Let $a_{j}, b_{k}, c$ denote the corresponding divisor classes so that $\Phi(A, B, C)=\chi(\widetilde{Y}, \mathcal{E})$ with

$$
\mathcal{E}=\mathcal{O}_{\widetilde{Y}}\left(\sum_{j} A_{j} a_{j}+\sum_{k} B_{k} b_{k}+C c\right) .
$$

We have $\mathbf{R} \pi_{*} \mathcal{O}_{\widetilde{Y}}=\mathcal{O}_{Y}$ where $\pi: \widetilde{Y} \rightarrow Y$ since $Y$ is nonsingular. So we first get

$$
\Phi(0,0, C)=\left(\begin{array}{c}
C+3 \\
3
\end{array}\right) .
$$

Applying the short exact sequence to an exceptional divisor which is isomorphic to $\widetilde{\mathbf{P}}^{2}$ and corresponds to some $b_{k}$, we get then inductively

$$
\Phi(0, B, C)=\sum_{k}\left(\begin{array}{c}
B_{k} \\
3
\end{array}\right)+\left(\begin{array}{c}
C+3 \\
3
\end{array}\right),
$$

using $\left(\begin{array}{c}B_{k} \\ 3\end{array}\right)-\left(\begin{array}{c}B_{k}-1 \\ 3\end{array}\right)=\left(\begin{array}{c}B_{k}-1 \\ 2\end{array}\right)=\left(\begin{array}{c}2-B_{k} \\ 2\end{array}\right)$. Indeed, the restriction of the exceptional divisor to itself is the hyperplane section class up to a sign using a general hyperplane of $\mathbf{P}^{3}$ 
passing through the point corresponding to $b_{k}$. Then we can use the same argument as above.

We apply the same argument to an exceptional divisor which is isomorphic to $\mathbf{P}^{1} \times \mathbf{P}^{1}$ and corresponds to some $a_{j}$. Let $n_{j}$ be the number of $b_{k}$ with $k \subset j$. Here we write $k \subset j$ when there is an inclusion between the corresponding $V$. Let $\mathbf{1}_{j}$ be as in the proof of Theorem (4.4). We have to calculate

$$
\Phi(A, B, C)-\Phi\left(A-\mathbf{1}_{j}, B, C\right)=\chi\left(E,\left.\mathcal{E}\right|_{E}\right)
$$

Let $e_{1}, e_{2}$ respectively denote the class of $p t \times \mathbf{P}^{1}$ and $\mathbf{P}^{1} \times p t$. Since the restrictions of $a_{j}$, $b_{k}(k \subset j)$ and $c$ are respectively

$$
\left(1-n_{j}\right) e_{1}-e_{2}, \quad e_{1}, \quad e_{1},
$$

the restriction of $\sum_{j} A_{j} a_{j}+\sum_{k} B_{k} b_{k}+C c$ to $\mathbf{P}^{1} \times \mathbf{P}^{1}$ is

$$
\left(\left(1-n_{j}\right) A_{j}+\sum_{k \subset j} B_{k}+C\right) e_{1}-A_{j} e_{2}
$$

Then

$$
\begin{aligned}
\chi\left(E,\left.\mathcal{E}\right|_{E}\right) & =\left(\left(1-n_{j}\right) A_{j}+\sum_{k \subset j} B_{k}+C+1\right)\left(1-A_{j}\right) \\
& =2\left(n_{j}-1\right)\left(\begin{array}{c}
A_{j} \\
2
\end{array}\right)-\left(A_{j}-1\right)\left(\sum_{k \subset j} B_{k}+C+1\right) .
\end{aligned}
$$

So we get

$$
\chi(\mathcal{E})=\sum_{j}\left(2\left(n_{j}-1\right)\left(\begin{array}{c}
A_{j}+1 \\
3
\end{array}\right)-\left(\begin{array}{c}
A_{j} \\
2
\end{array}\right)\left(\sum_{k \subset j} B_{k}+C+1\right)\right)+\sum_{k}\left(\begin{array}{c}
B_{k} \\
3
\end{array}\right)+\left(\begin{array}{c}
C+3 \\
3
\end{array}\right) .
$$

We apply this to $\mathcal{E}$ with $A_{j}=2-\left\lceil i m_{j} / d\right\rceil, B_{j}=3-\left\lceil i m_{j} / d\right\rceil, C=i-4$, where $i=d-k$ and $p=3$ in Proposition (1.5), see (1.4.5). Then Theorem 4 follows.

\section{Proofs of Theorems 1-4 by HRR}

5.1. Hirzebruch-Riemann-Roch Theorem. For a vector bundle $\mathcal{E}$ of rank $r$ on a smooth complex projective variety $X$, there are Chern classes $c_{i}(\mathcal{E}) \in H^{2 i}(X, \mathbf{Q})$ such that $c_{0}(\mathcal{E})=1, c_{i}(\mathcal{E})=0$ for $i>r$, and the following facts are well known (see [17], [19]):

(a) The total Chern class, the Chern character, and the Todd class are defined by

$$
c(\mathcal{E})=\sum_{i} c_{i}(\mathcal{E}), \quad c h(\mathcal{E})=\sum_{1 \leq i \leq r} \exp \left(x_{i}\right), \quad \operatorname{Td}(\mathcal{E})=\prod_{1 \leq i \leq r} Q\left(x_{i}\right),
$$

where $Q(x)=x /(1-\exp (-x))$ and the formal Chern roots $x_{i}$ satisfy

$$
\prod_{1 \leq i \leq r}\left(1+x_{i} t\right)=\sum_{i} c_{i}(\mathcal{E}) t^{i}
$$

(b) The total Chern class and the Todd class of $X$ are defined by

$$
c(X)=c\left(T_{X}\right), \quad T d(X)=T d\left(T_{X}\right) .
$$


(c) By the Hirzebruch-Riemann-Roch theorem [19] we have

$$
\chi(\mathcal{E})=\int_{X} \operatorname{ch}(\mathcal{E}) \operatorname{Td}(X)
$$

We will need the following properties of the characteristic classes:

(d) For a short exact sequence of vector bundles $0 \rightarrow \mathcal{E}^{\prime} \rightarrow \mathcal{E} \rightarrow \mathcal{E}^{\prime \prime} \rightarrow 0$, we have

$$
c(\mathcal{E})=c\left(\mathcal{E}^{\prime}\right) c\left(\mathcal{E}^{\prime \prime}\right), \quad \operatorname{ch}(\mathcal{E})=\operatorname{ch}\left(\mathcal{E}^{\prime}\right)+\operatorname{ch}\left(\mathcal{E}^{\prime \prime}\right), \quad \operatorname{Td}(\mathcal{E})=\operatorname{Td}\left(\mathcal{E}^{\prime}\right) \operatorname{Td}\left(\mathcal{E}^{\prime \prime}\right) .
$$

(e) For the tensor product of two vector bundles $\mathcal{E}, \mathcal{F}$ we have

$$
\operatorname{ch}(\mathcal{E} \otimes \mathcal{F})=\operatorname{ch}(\mathcal{E}) \operatorname{ch}(\mathcal{F})
$$

(f) For the exterior product we have

$$
\sum_{i} c_{i}\left(\bigwedge^{p} \mathcal{E}\right) t^{i}=\prod_{i_{1}<\cdots<i_{p}}\left(1+\left(x_{i_{1}}+\cdots x_{i_{p}}\right) t\right) .
$$

(g) For the dual vector bundle $\mathcal{E}^{\vee}$, we have

$$
c_{i}\left(\mathcal{E}^{\vee}\right)=(-1)^{i} c_{i}(\mathcal{E}) .
$$

5.2. Remarks. (i) The function $Q(x)$ has the expansion

$$
Q(x)=1+\frac{1}{2} x+\sum_{k=1}^{\infty}(-1)^{k-1} \frac{B_{k}}{(2 k) !} x^{2 k},
$$

where the $B_{k}$ are the Bernoulli numbers, see [17], Ex. 3.2.4. The first few terms of $B_{k}$ are

$$
\frac{1}{6}, \frac{1}{30}, \frac{1}{42}, \frac{1}{30}, \frac{5}{66}, \ldots
$$

(ii) Using $c_{i}=c_{i}(\mathcal{E})$ and $r=\operatorname{rank} \mathcal{E}$, we have the expansions (see [17], Ex. 3.2.3-4)

$$
\begin{aligned}
\operatorname{ch}(\mathcal{E}) & =r+c_{1}+\frac{1}{2}\left(c_{1}^{2}-2 c_{2}\right)+\frac{1}{6}\left(c_{1}^{3}-3 c_{1} c_{2}+c_{3}\right)+\cdots, \\
T d(\mathcal{E}) & =1+\frac{1}{2} c_{1}+\frac{1}{12}\left(c_{1}^{2}+c_{2}\right)+\frac{1}{24}\left(c_{1} c_{2}\right)+\cdots
\end{aligned}
$$

(iii) By $(5.1 .2), c(\mathcal{E}), \operatorname{ch}(\mathcal{E}), \operatorname{Td}(\mathcal{E})$ are extended to well-defined morphisms

$$
c(\mathcal{E}), \operatorname{ch}(\mathcal{E}), \operatorname{Td}(\mathcal{E}): K^{0}(X) \rightarrow H^{\bullet}(X, \mathbf{Q}),
$$

where the source is the Grothendieck group of vector bundles on $X$. Note that the initial term of $c h(\mathcal{E})$ is the virtual rank of $\mathcal{E}$, and the latter does not appear in $c(\mathcal{E}), T d(\mathcal{E})$. 
(iv) For $n=\operatorname{dim} X$ we have

$$
\int_{X} c_{n}(X)=\chi(X, \mathbf{C}), \quad \int_{X} \operatorname{Td}(X)_{n}=\chi\left(X, \mathcal{O}_{X}\right),
$$

where $\chi(X, \mathbf{C})$ is the topological Euler characteristic of $X$. For the first assertion, see e.g. [17], Ex. 8.1.12. The second assertion follows from the Hirzebruch-Riemann-Roch theorem (5.1.1) applied to $\mathcal{E}=\mathcal{O}_{X}$ where $c_{i}(\mathcal{E})=0$ for $i>0$.

5.3. Combinatorial description of the cohomology. Let $D$ be an essential central hyperplane arrangement. In the notation of (1.1) we apply the construction in (2.1) to

$$
\mathcal{S}:=\mathcal{S}(D)^{\mathrm{nnc}},
$$

and not to $\mathcal{S}(D)$ as in [4], [5]. (This simplifies some arguments in loc. cit. considerably.) Note that $\gamma(V) \geq 2$ for $V \in \mathcal{S}$. By C. De Concini and C. Procesi [8] the cohomology ring of $\widetilde{Y}$ in (2.1) is described by using only the combinatorial data as follows:

Let $\mathbf{Q}\left[e_{V}\right]_{V \in \mathcal{S}}$ be the polynomial ring with independent variables $e_{V}$ for $V \in \mathcal{S}$. There is an isomorphism

$$
\mathbf{Q}\left[e_{V}\right]_{V \in \mathcal{S}} / I_{\mathcal{S}} \stackrel{\sim}{\longrightarrow} H^{\bullet}(\widetilde{Y}, \mathbf{Q}),
$$

sending $e_{V}$ to $\left[E_{V}\right]$ for $V \neq 0$ and $e_{0}$ to $-\left[E_{0}\right]$, where $E_{0}$ is the total (or proper) transform of a general hyperplane which was denoted by $\widetilde{H}$. Moreover, the ideal $I_{\mathcal{S}}$ is generated by

$$
R_{V, W}= \begin{cases}e_{V} e_{W} & \text { if } V, W \text { are incomparable, } \\ e_{V} \widetilde{e}_{W}^{\gamma(W)-\gamma(V)} & \text { if } W \varsubsetneqq V, \\ \widetilde{e}_{W}^{\gamma(W)} & \text { if } V=\mathbf{C}^{n},\end{cases}
$$

where $\widetilde{e}_{W}:=\sum_{W^{\prime} \subset W} e_{W^{\prime}}$ and $\gamma(V):=\operatorname{codim} V$. Here $V, W, W^{\prime} \in \mathcal{S}$ except for the third case where $V=\mathbf{C}^{n}$. Note that $\mathcal{S}$ is stable by intersection so that a nested subset of $\mathcal{S}$ in the sense of $[8]$ is always linearly ordered by the inclusion relation.

For $V \in \mathcal{S}(D) \backslash \mathcal{S}(D)^{\mathrm{nnc}}$, let $\mathbf{P}(V)^{\sim}$ denote the proper transform of $\mathbf{P}(V)$ in $\tilde{Y}$. (Here the notation $\mathbf{P}(V)^{\mathcal{S}^{V}}$ in Section 2 cannot be used since $V \notin \mathcal{S}:=\mathcal{S}(D)^{\mathrm{nnc}}$.) Then the cohomology class $e_{V}$ of $\mathbf{P}(V)^{\sim}$ is given by $\prod_{D_{j} \supset V} e_{D_{j}}$ since $\mathbf{P}(V)^{\sim}$ is the intersection of $\mathbf{P}\left(D_{j}\right)^{\sim}$ with $D_{j} \supset V$. For $V=D_{j}$, we have by calculating the total transform of $D_{j}$

$$
e_{D_{j}}+\sum_{W \in \mathcal{S}, W \subset D_{j}} e_{W}=0,
$$

since $e_{0}$ corresponds to $-\widetilde{H}$. (This is similar to (5.3.2) for $\mathcal{S}=\mathcal{S}(D)$ although $\widetilde{Y}$ is different.)

5.4. Calculation of Chern classes. In our case the Chern classes of $\widetilde{Y}$ are expressed by applying inductively the formula for the Chern classes under the blow-up ([17], 15.4). By [4] we have $c(\widetilde{Y})=\prod_{V \in \mathcal{S}} F_{V}$, under the isomorphism (5.3.1), with

$$
F_{V}= \begin{cases}\left(1-\widetilde{e}_{V}^{\prime}\right)^{-\gamma(V)}\left(1+e_{V}\right)\left(1-\widetilde{e}_{V}\right)^{\gamma(V)} & \text { if } V \neq 0, \\ \left(1-e_{0}\right)^{n} & \text { if } V=0,\end{cases}
$$


where $\widetilde{e}_{V}:=\sum_{W \subset V} e_{W}, \widetilde{e}_{V}^{\prime}:=\widetilde{e}_{V}-e_{V}$, and $\gamma(V):=\operatorname{codim} V$. Using the Grothendieck group as in $(5.2 .3),(5.4 .1)$ implies that $\operatorname{Td}(\widetilde{Y})=\prod_{V \in \mathcal{S}} G_{V}$ with

$$
G_{V}= \begin{cases}Q\left(-\widetilde{e}_{V}^{\prime}\right)^{-\gamma(V)} Q\left(e_{V}\right) Q\left(-\widetilde{e}_{V}\right)^{\gamma(V)} & \text { if } V \neq 0 \\ Q\left(-e_{0}\right)^{n} & \text { if } V=0\end{cases}
$$

So the Chern classes and the Todd class of $\widetilde{Y}$ are expressed by using only the combinatorial data via (5.3.1).

Set $\mathcal{S}^{\prime}=\mathcal{S}(D)^{\text {nnc }} \cup\left\{D_{i}\right\}$ where the $D_{i}$ are the irreducible components of $D$. The proper transform $\mathbf{P}\left(D_{i}\right)^{\sim}$ of $\mathbf{P}\left(D_{i}\right)$ in $\tilde{Y}$ will be denoted by $E_{D_{i}}$. By (5.3.3) its cohomology class $e_{D_{i}}$ is given by

$$
e_{D_{i}}=-\sum_{W \in \mathcal{S}, W \subset D_{i}} e_{W} \in \mathbf{Q}\left[e_{W}\right]_{W \in \mathcal{S}} / I_{\mathcal{S}}
$$

We have a short exact sequence

$$
0 \rightarrow \Omega_{\widetilde{Y}}^{1} \rightarrow \Omega_{\widetilde{Y}}^{1}(\log \widetilde{Z}) \rightarrow \bigoplus_{V \in \mathcal{S}^{\prime} \backslash\{0\}} \mathcal{O}_{E_{V}} \rightarrow 0
$$

Using (5.2.3), we get then

$$
c\left(\Omega_{\widetilde{Y}}^{1}(\log \widetilde{Z})\right)=c\left(\Omega_{\widetilde{Y}}^{1}\right) \prod_{V} c\left(\mathcal{O}_{E_{V}}\right)=c\left(\Omega_{\widetilde{Y}}^{1}\right) \prod_{V} c\left(\mathcal{O}_{\widetilde{Y}}\left(-E_{V}\right)\right)^{-1} .
$$

Moreover, the Chern classes of $\Omega_{\widetilde{Y}}^{p}(\log \widetilde{Z})=\bigwedge^{p} \Omega_{\widetilde{Y}}^{1}(\log \widetilde{Z})$ for $p>1$ are expressed by using those of $\Omega_{\widetilde{Y}}^{1}(\log \widetilde{Z})$ by $(5.1 .4)$. (However, it is not easy to write down the universal polynomials explicitly.)

5.5. Proofs of Theorems 1-2 by HRR. We calculate the right-hand side of (1.5.1) in Proposition (1.5) by applying the Hirzebruch-Riemann-Roch theorem (5.1.1) to

$$
\mathcal{E}_{k}=\Omega_{\widetilde{Y}}^{p}(\log \widetilde{Z}) \otimes_{\mathcal{O}} \mathcal{O}_{\widetilde{Y}}\left(-k \widetilde{H}+\sum_{j}\left\lfloor k m_{j} / d\right\rfloor E_{j}\right)
$$

where the $m_{j}$ are given by $\mu(V)$ if $E_{j}$ in Proposition (1.5) is $E_{V}$ in (2.1). For $\mathcal{O}_{\widetilde{Y}}\left(D_{k}\right)$ with

$$
D_{k}=-k \widetilde{H}+\sum_{j}\left\lfloor k m_{j} / d\right\rfloor E_{j}
$$

we have $c\left(\mathcal{O}_{\widetilde{Y}}\left(D_{k}\right)\right)=1+\left[D_{k}\right]$. Then we can apply (5.1.3) to calculate $\operatorname{ch}\left(\mathcal{E}_{k}\right)$, and $\chi\left(\mathcal{E}_{k}\right)$ depends only on the combinatorial data using the assertions in (5.4) together with the Hirzebruch-Riemann-Roch theorem (5.1.1). In the reduced case the $n_{f, \alpha}$ depend only on the combinatorial data as in Theorem 2. Moreover, if $p=0$ or $p=n-1$, then $n_{f, \alpha}$ for $\alpha \in(0,1] \cup(n-1, n)$ depends only on the weak equivalence class using (1.4.5) for $p=n-1$ since $\Omega_{\widetilde{Y}}^{0}(\log \widetilde{Z})=\mathcal{O}_{\widetilde{Y}}$ for $p=0$. So the assertion follows.

5.6. Remark. We can prove Theorem (4.4) by using (5.3-4), and this is enough for the proofs of Theorems 1-2 as is shown in (4.5).

In the following, we illustrate how to calculate $n_{f, \alpha}$ using this method. 
5.7. Proof of Theorem 3 by HRR. Let $a_{i}$ denote the $e_{V} \bmod I_{\mathcal{S}}$ in $(5.3)$ for $V \in \mathcal{S}^{(2)}$ (see $(1.1 .3)$ ), i.e. the $a_{i}$ correspond to the points of $\mathbf{P}\left(D^{\mathrm{nnc}}\right)$. Set $c=e_{0}$. We have the relations

$$
a_{i} a_{j}=0(i \neq j), a_{i} c=0, a_{i}^{2}=-c^{2}, c^{3}=0,
$$

using $\left(a_{i}+c\right)^{2}=0$, etc. in (5.3.2). Let $F_{i}^{\prime}$ denote $F_{V}$ for $V$ corresponding to $a_{i}$. Then

$$
F_{i}^{\prime}=(1-c)^{-2}\left(1+a_{i}\right)\left(1-c-a_{i}\right)^{2}=1-a_{i}+c^{2}
$$

and $F_{0}=(1-c)^{3}$. Set $\nu^{(2)}=\sum_{m \geq 3} \nu_{m}^{(2)}$ with $\nu_{m}^{(2)}$ as in (1.1.3). Since $c(\tilde{Y})=F_{0} \prod_{i} F_{i}^{\prime}$, we get

$$
c(\tilde{Y})=1-\left(\sum a_{i}+3 c\right)+\left(\nu^{(2)}+3\right) c^{2}, \quad \operatorname{Td}(\tilde{Y})=1-\frac{1}{2}\left(\sum a_{i}+3 c\right)+c^{2},
$$

using (5.2.2). Note that $\Omega_{\widetilde{Y}}^{2}=\mathcal{O}_{\widetilde{Y}}\left(-3 \widetilde{H}+\sum_{i} E_{i}\right)$ where the $E_{i}$ correspond to $a_{i}$. So the Hirzebruch-Riemann-Roch theorem for a line bundle coincides with the Riemann-Roch theorem for surfaces, and the argument is the same as in (3.1) if $p=2$ or 0 .

In (3.1), the assertion for $p=1$ is reduced to the other cases using the relation with $\chi(U)$. However, it should be possible to prove it by using the Hirzebruch-Riemann-Roch theorem for vector bundles. We apply this to

$$
\mathcal{E}_{k}=\Omega_{\widetilde{Y}}^{1}(\log \widetilde{Z}) \otimes \mathcal{O}_{\widetilde{Y}}\left(D_{k}\right),
$$

with $D_{k}=-k \widetilde{H}+\sum_{j}\left\lfloor k m_{j} / d\right\rfloor E_{j}$. By the calculation of $c(\widetilde{Y})$ together with (5.1.5) we have

$$
c\left(\Omega_{\widetilde{Y}}^{1}\right)=1+\sum a_{i}+3 c+\left(\nu^{(2)}+3\right) c^{2} .
$$

So we get by $(5.4 .3)$

$$
c\left(\Omega_{\widetilde{Y}}^{1}(\log \widetilde{Z})\right)=\left(1+\sum a_{i}+3 c+\left(\nu^{(2)}+3\right) c^{2}\right) \prod_{i}\left(1-a_{i}\right)^{-1} \prod_{j}\left(1-a_{j}^{\prime}\right)^{-1} .
$$

Here $a_{j}^{\prime}:=-\sum_{i \subset j} a_{i}-c$ which corresponds to the proper transform of an irreducible component $\mathbf{P}\left(D_{j}\right)$ of $\mathbf{P}(D)$, and we write $i \subset j$ if the corresponding subspaces of $\mathbf{P}^{2}$ have such an inclusion relation. (Note that $-c$ corresponds to $\widetilde{H}$.) We have

$$
\prod_{j}\left(1+\sum_{i \subset j} a_{i}+c\right)=1+\sum_{i} m_{i} a_{i}+d c+\left(\left(\begin{array}{c}
d \\
2
\end{array}\right)-\sum_{i}\left(\begin{array}{c}
m_{i} \\
2
\end{array}\right)\right) c^{2},
$$

and $c\left(\Omega_{\widetilde{Y}}^{1}(\log \widetilde{Z})\right)$ is equal to

$$
1+\sum_{i}\left(2-m_{i}\right) a_{i}+(3-d) c-\frac{1}{2}\left(\left(d^{2}-5 d+2 \nu^{(2)}+6\right)-\sum_{i}\left(m_{i}^{2}-3 m_{i}+4\right)\right) c^{2} .
$$

Then $\operatorname{Td}(\widetilde{Y}), \frac{1}{2} \operatorname{ch}\left(\Omega_{\widetilde{Y}}^{1}(\log \widetilde{Z})\right), \operatorname{ch}\left(\mathcal{O}_{\widetilde{Y}}\left(D_{k}\right)\right)$ are respectively

$$
\begin{aligned}
& 1-\frac{1}{2}\left(\sum_{i} a_{i}+3 c\right)+c^{2}, \\
& 1-\frac{1}{2}\left(\sum_{i}\left(m_{i}-2\right) a_{i}+(d-3) c\right)+\frac{1}{4}\left(\sum_{i} m_{i}-d-2 \nu^{(2)}+3\right) c^{2}, \\
& 1+\left(\sum_{i}\left\lfloor k m_{i} / d\right\rfloor a_{i}+k c\right)-\frac{1}{2}\left(\sum_{i}\left\lfloor k m_{i} / d\right\rfloor^{2}-k^{2}\right) c^{2} .
\end{aligned}
$$


Calculating the degree 2 part of the multiplication of these three, we get the right-hand side of the second equation divided by -2 in Theorem 3 where $i=d-k$.

5.8. Proof of Theorem 4 by HRR. Let $a_{j}\left(j \in I^{\prime}\right), b_{k}\left(k \in I^{\prime \prime}\right), c$ denote the $e_{V} \bmod$ $I_{\mathcal{S}}$ in (5.3) for $V \in \mathcal{S}^{(i)}$ (see (1.1.3)) depending on whether $i=2$ or 3 or 4 . We will write $k \subset j$ if there is an inclusion relation between the corresponding $V$. Let $n_{j}\left(j \in I^{\prime}\right)$ be the number of $b_{k}\left(k \in I^{\prime \prime}\right)$ with $k \subset j$. We have the relations

$$
\begin{aligned}
& a_{i} a_{j}=b_{k} b_{l}=a_{j} b_{k}^{2}=a_{j} c^{2}=b_{k} c=0(i \neq j, k \neq l), a_{j} b_{k}=0(k \not \subset j), \\
& a_{j} b_{k}=-a_{j} c(k \subset j), a_{j}^{3}=-2\left(n_{j}-1\right) c^{3}, a_{j}^{2} c=b_{k}^{3}=-c^{3}, a_{j}^{2} b_{k}=c^{3}(k \subset j),
\end{aligned}
$$

using $\left(a_{j}+\sum_{k \subset j} b_{k}+c\right)^{2}=0,\left(b_{k}+c\right)^{3}=0, a_{j}\left(b_{k}+c\right)=0(k \subset j), a_{j} c^{2}=0$, see (5.3.2). By the same argument as in $(4.8)$ it is enough to calculate $\chi(\mathcal{E})$ for a line bundle $\mathcal{E}$ with

$$
c_{1}(\mathcal{E})=\sum_{j} A_{j} a_{j}+\sum_{k} B_{k} b_{k}+C c\left(A_{j}, B_{k}, C \in \mathbf{Z}\right)
$$

Let $F_{j}^{\prime}, F_{k}^{\prime \prime}$ denote $F_{V}$ if $V$ corresponds to $a_{j}, b_{k}$ respectively. Then

$$
F_{j}^{\prime}=1-a_{j}-a_{j}^{2}+2\left(n_{j}-1\right) a_{j} c-2\left(n_{j}-1\right) c^{3}, \quad F_{k}^{\prime \prime}=1-2 b_{k}-2 c^{3} .
$$

Since $c(\widetilde{Y})=F_{0} \prod_{j} F_{j}^{\prime} \prod_{k} F_{k}^{\prime \prime}$ with $F_{0}=(1-c)^{4}$, we get

$$
c_{1}(\tilde{Y})=-\sum_{j} a_{j}-\sum_{k} 2 b_{k}-4 c, \quad c_{2}(\tilde{Y})=\sum_{j}\left(2 a_{j} c-a_{j}^{2}\right)+6 c^{2}
$$

where $c_{3}(\tilde{Y})$ is the topological Euler characteristic $\chi(\widetilde{Y})$ multiplied by $-c^{3}$, see (5.2.4). This gives $\operatorname{Td}(\widetilde{Y})$ using the expansion of the Todd class in (5.2.2) (where $c_{3}$ does not appear so that $c_{3}(\widetilde{Y})$ is not needed). Thus we get

$$
\begin{aligned}
& \operatorname{Td}(\tilde{Y})_{1}=-\sum_{j} \frac{a_{j}}{2}-\sum_{k} b_{k}-2 c \\
& \operatorname{Td}(\tilde{Y})_{2}=\sum_{j} \frac{\left(5-2 n_{j}\right) a_{j} c}{6}+\sum_{k} \frac{b_{k}^{2}}{3}+\frac{11 c^{2}}{6}
\end{aligned}
$$

where $\operatorname{Td}(\widetilde{Y})_{3}=-c^{3}$, see (5.2.4). We apply this theorem to the line bundle $\mathcal{E}$ in $(5.8 .1)$. Then we get

$$
\chi(\mathcal{E})=\sum_{j}\left(2\left(n_{j}-1\right)\left(\begin{array}{c}
A_{j}+1 \\
3
\end{array}\right)-\left(\begin{array}{c}
A_{j} \\
2
\end{array}\right)\left(\sum_{k \subset j} B_{k}-C+1\right)\right)+\sum_{k}\left(\begin{array}{c}
B_{k} \\
3
\end{array}\right)-\left(\begin{array}{c}
C-1 \\
3
\end{array}\right),
$$

which is compatible with (4.8.1) where $C$ corresponds to $-C$. We apply this to $\mathcal{E}$ with $A_{j}=2-\left\lceil i m_{j} / d\right\rceil, B_{j}=3-\left\lceil i m_{j} / d\right\rceil, C=4-i$, where $i=d-k$ and $p=3$ in Proposition (1.5), see (1.4.5). Then Theorem 4 follows. 


\section{References}

[1] Beilinson, A., Bernstein, J. and Deligne, P., Faisceaux pervers, Astérisque, vol. 100, Soc. Math. France, Paris, 1982.

[2] Brieskorn, E., Sur les groupes de tresses [d'après V.I. Arnold], Séminaire Bourbaki, 24ème année (1971/1972), Exp. No. 401, Lect. Notes in Math. Vol. 317, Springer, Berlin, 1973, pp. 21-44.

[3] Budur, N., On Hodge spectrum and multiplier ideals, Math. Ann. 327 (2003), 257-270.

[4] Budur, N., Jumping numbers of hyperplane arrangements, arXiv:0802.0878 to appear in Comm. Algebra.

[5] Budur, N., Hodge spectrum of hyperplane arrangements, arXiv:0809.3443 (unpublished).

[6] Budur, N. and Saito, M., Multiplier ideals, $V$-filtration, and spectrum, J. Alg. Geom. 14 (2005), 269-282.

[7] Cohen, D.C. and Suciu, A., On Milnor fibrations of arrangements, J. London Math. Soc. 51 (1995), 105-119.

[8] De Concini, C. and Procesi, C., Wonderful models of subspace arrangements, Selecta Math. (N.S.) 1 (1995), 459-494.

[9] Deligne, P., Equations Différentiellesà Points Singuliers Réguliers, Lect. Notes in Math. vol. 163, Springer, Berlin, 1970.

[10] Deligne, P., Théorie de Hodge II, Publ. Math. IHES, 40 (1971), 5-58.

[11] Deligne, P., Le formalisme des cycles évanescents, in SGA7 XIII and XIV, Lect. Notes in Math. 340, Springer, Berlin, 1973, pp. 82-115 and 116-164.

[12] Dimca, A., Singularities and Topology of Hypersurfaces, Universitext, Springer, Berlin, 1992.

[13] Dimca, A. and Saito, M., A generalization of Griffiths's theorem on rational integrals, Duke Math. J. 135 (2006), 303-326.

[14] Ein, L., Lazarsfeld, R., Smith, K.E. and Varolin, D., Jumping coefficients of multiplier ideals, Duke Math. J. 123 (2004), 469-506.

[15] Esnault, H., Fibre de Milnor d'un cône sur une courbe plane singulière, Inv. Math. 68 (1982), 477-496.

[16] Esnault, H. and Viehweg, E., Revêtements cycliques, in Algebraic threefolds (Varenna, 1981), Lecture Notes in Math., 947, Springer, Berlin-New York, 1982, pp. 241-250.

[17] Fulton, W., Intersection Theory, Springer, Berlin, 1984.

[18] Hartshorne, R., Algebraic Geometry, Springer, Berlin, 1977.

[19] Hirzebruch, F., Topological method in algebraic geometry, Springer, Berlin, 1966.

[20] Jouanolou, J.-P., Cohomologie de quelques schémas classiques et théorie cohomologique des classes de Chern, in SGA5, Lecture Notes in Math. 589, Springer, Berlin, 1977, pp. 282-350.

[21] Lazarsfeld, R., Positivity in algebraic geometry II, Ergebnisse der Mathematik und ihrer Grenzgebiete. 3. Folge, A series of Modern Surveys in Mathematics, Vol. 49, Springer-Verlag, Berlin, 2004.

[22] Mustaţă, M., Multiplier ideals of hyperplane arrangements, Trans. Amer. Math. Soc. 358 (2006), 5015-5023.

[23] Nadel, A.M., Multiplier ideal sheaves and Kähler-Einstein metrics of positive scalar curvature, Ann. Math. 132 (1990), 549-596.

[24] Orlik, P. and Solomon, L., Combinatorics and topology of complements of hyperplanes, Inv. Math. 56 (1980), 167-189. 
[25] Rybnikov, G., On the fundamental group of the complement of a complex hyperplane arrangement (math.AG/9805056).

[26] Saito, M., Mixed Hodge modules, Publ. RIMS, Kyoto Univ. 26 (1990), 221-333.

[27] Saito, M., Multiplier ideals, $b$-function, and spectrum of a hypersurface singularity, Compos. Math. 143 (2007) 1050-1068.

[28] Saito, M., Bernstein-Sato polynomials of hyperplane arrangements (math.AG/0602527).

[29] Saito, M., Jumping coefficients and spectrum of a hyperplane arrangement (unpublished manuscript, 2007).

[30] Schechtman, V., Terao, H. and Varchenko, A., Local systems over complements of hyperplanes and the Kac-Kazhdan conditions for singular vectors, J. Pure Appl. Algebra 100 (1995), 93-102.

[31] Steenbrink, J.H.M., The spectrum of hypersurface singularity, Astérisque 179-180 (1989), 163-184.

[32] Teitler, Z., A note on Mustaţă's computation of multiplier ideals of hyperplane arrangements, Proc. Amer. Math. Soc. 136 (2008), 1575-1579.

[33] Walther, U., Bernstein-Sato polynomial versus cohomology of the Milnor fiber for generic hyperplane arrangements, Compos. Math. 141 (2005), 121-145.

Department of Mathematics, The University of Notre Dame, IN 46556, USA

e-mail: nbudur@nd.edu

RIMS Kyoto University, Kyoto 606-8502 Japan

e-mail: msaito@kurims.kyoto-u.ac.jp

Aug. 26, 2009, v.3 